\title{
FLORA PALINOLÓGICA DE LOS HELECHOS Y LICOFITAS de Río Malila, Hidalgo, México
}

\author{
Felipe Gómez-Noguez ${ }^{1}{ }^{13}$, Blanca Pérez-García ${ }^{1}$, Aniceto Mendoza-Ruiz ${ }^{1}$ \\ y Alma Orozco-SeGovia ${ }^{2}$ \\ 'Departamento de Biología, División de Ciencias Biológicas y de la Salud, \\ Universidad Autónoma Metropolitana-Iztapalapa, México, D.F., México \\ ${ }^{2}$ Departamento de Ecología Funcional, Instituto de Ecología, \\ Universidad Nacional Autónoma de México, Ciudad Universitaria, México, D.F., México \\ ${ }_{3}^{3}$ Autor para la correspondencia: fgnoguez@ciencias.unam.mx
}

\begin{abstract}
Resumen: Se presentan datos relativos a la morfología de las esporas de 36 especies de helechos y tres licofitas presentes en la localidad de Río Malila, municipio de Molango de Escamilla, en el estado de Hidalgo, México. Los taxa estudiados corresponden a 27 géneros y 14 familias. Las esporas de Ctenitis equestris, Elaphoglossum obscurum, Pecluma sursumcurrens y Polystichum turrialbae se describen por primera vez. Las descripciones palinológicas se realizaron con base en observaciones de material fresco y con microscopía de luz y/o electrónica de barrido y se incluyen imágenes para cada uno de los taxa estudiados. Como resultado se elaboró una clave dicotómica para la identificación de las especies presentes en la zona a partir de sus esporas. El conocimiento palinológico de los helechos puede contribuir a resolver problemas taxonómicos del grupo, ayudar en el diagnóstico oportuno de poblaciones en riesgo y facilitar posteriores estudios aerobiológicos de las pteridofitas, desde la identificación de esporas presentes en el aire, la determinación de patrones fenológicos de comunidades, la dispersión de esporas en diferentes condiciones meteorológicas y ambientales, hasta su posible efecto en la salud de otros organismos.
\end{abstract}

Palabras clave: esporas, MEB, microscopia óptica, morfología, palinología.

\begin{abstract}
Morphological characteristics of the spores of 36 species of ferns and three of lycophytes from Rio Malila, municipality of Molango de Escamilla, Hidalgo, Mexico, grouped into 27 genera and 14 families are provided. The spores of Ctenitis equestris, Elaphoglossum obscurum, Polystichum turrialbae, and Pecluma sursumcurrens are described for the first time. Palynological descriptions were based on observations of fresh material with light microscopy and/or scanning electron microscopy, images are included for each species. As a result dichotomous key for the identification of all species studied was elaborated. The palynologic knowledge of the ferns may contribute to resolve taxonomic problems in the group, to the opportune diagnose of risk populations and to facilitate aerobiological studies of the pteridophytes, like the airborne spore identification, the dispersal of spores in different weather and environmental conditions, and its possible health risk for other organisms.
\end{abstract}

Key words: light microscopy, morphology, palynology, SEM, spore.

$\mathbf{L}$ a mayoría de los estudios palinológicos han centrado su atención sobre los granos de polen y por ende se tiene un mayor conocimiento de éstos que de las esporas de los helechos (Tryon y Lugardon, 1991). Esto quizás se debe a sus efectos en la salud humana (polinosis), así como a su aplicación en las prácticas de cultivo agrícola y ornamental (Benito-Rica y Soto-Torres, 2001). Sin embargo, desde mediados del siglo XX se han realizado investigaciones sobre la morfología de esporas de helechos en diversas partes del mundo, pues son reconocidos como buenos microfósiles y su conocimiento provee evidencias de la diversificación temprana de las plantas vasculares; así como de cambios climáticos y de vegetación (Tryon y Lugardon, 1991). Además, cada taxon puede ser identificado palinológicamente con un buen nivel de precisión (Large y Braggins, 1991). Algunos de estos estudios abordan el tema bajo diferentes enfoques, desde trabajos de floras locales (Tschudy y Tschudy, 1965; Large y Braggins, 1991; Contreras-Duarte et 
al., 2006), pasando por el tratamiento de familias, géneros o grupos taxonómicos particulares (Hagenah, 1961; Britton y Jeremy, 1974; Morbelli, 1976; Pangua y Prada, 1988; Morbelli y Michelena, 1989; Giudice et al., 2004, 2006; Morbelli y Giudice, 2010) e investigaciones de otra índole, incluyendo aspectos de fisiología y ecología (Lugardon, 1986; Tryon, 1986), hasta estudios de morfogénesis (Uhera y Kurita, 1986). En México sobresalen los trabajos realizados en el valle de México (Arreguín-Sánchez, 1985, 1988, 1991); Nuevo León (Arreguín-Sánchez y Aguirre-Claverán, 1990) y Querétaro (Arreguín-Sánchez et al., 1996); así como las contribuciones del proyecto Morfogénesis de la fase sexual de helechos (Pérez-García et al., 1995; Mendoza-Ruiz, 2001; Pérez-García y Mendoza-Ruiz, 2005; Mendoza-Ruiz y Pérez-García, 2009; Pérez-García et al., 2010). Por su parte, Prada (1990) ha realizado una revisión general y completa de diversos aspectos relacionados con el estudio de las esporas de helechos, quien menciona una variación de hasta $30 \%$ en el tamaño reportado para diversas especies dependiendo de la técnica empleada, siendo mayores las esporas en los trabajos en los cuales se aplica la acetólisis de Erdtman (1960), que en las reportadas sin esta técnica. Menciona también que dicha técnica puede provocar el desprendimiento, la fragmentación o la destrucción del perisporio, lo que en ocasiones dificulta la identificación de las especies.

Los estudios morfológicos de las esporas de helechos han permitido tener un mejor conocimiento de la sistemática en algunas familias de helechos como Cyatheaceae (Gastony, 1979) y Thelypteridaceae (Wood, 1973), así como la realización de otro tipo de investigaciones aerobiológicas para determinar la concentración de esporas en sitios urbanos (Lacey y McCartney, 1994) y sus repercusiones en la salud del hombre (Chew et al., 2000; Ching, 2004). El presente estudio tuvo como propósito contribuir al conocimiento palinológico de la flora mexicana y facilitar información que resulte de utilidad para estudios posteriores de lluvia de esporas.

\section{Materiales y métodos}

Área de estudio. Todo el material biológico utilizado en este trabajo fue obtenido en un bosque mesófilo del estado de Hidalgo, cuya pteridoflora no ha sido estudiada a cabalidad (Ramírez-Cruz et al., 2009). Se seleccionó la localidad de Río Malila, la cual se encuentra próxima al km 118 de la carretera federal 105 Pachuca-Tampico, enclavada en la Sierra Madre Oriental. El área de estudio se encuentra ubicada en los $20^{\circ} 43^{\prime} 21^{\prime \prime}$ de latitud $\mathrm{N}$ y $98^{\circ} 43^{\prime} 36^{\prime \prime}$ de longitud O, a una altitud de 1,507 m s.n.m., pertenece al municipio de Molango de Escamilla, en donde predomina el clima C(fm) b(e)g (templado húmedo con lluvias todo el año) y el tipo de suelo es luvisol órtico y cambisol éutrico y los afloramientos rocosos corresponden a rocas sedimentarias (calizas y lutitas) del Jurásico superior (Ramírez-Trejo, 2002).
Río Malila se localiza en la subprovincia Carso-Huasteco y el tipo de vegetación presente en la zona corresponde al bosque mesófilo de montaña, caracterizado por la presencia de Liquidambar styraciflua L. y varias especies del género Quercus L. que alcanzan los 35 metros de altura; sin embargo, la actividad agrícola ha provocado una disminución de la cobertura, perceptible por la presencia de manchones de vegetación en buen estado de conservación, en las zonas de cañadas y de pendientes escarpadas.

Recolección de material biológico y procesamiento de muestras. Se realizaron 14 salidas al campo en diferentes épocas del año, entre los meses de febrero del 2009 y marzo del 2010 y se recolectaron 74 ejemplares de helechos y licofitas, de acuerdo con las técnicas descritas por Lorea y Riba (1990). Cada ejemplar se determinó, utilizando las claves de identificación incluidas en la obra de Mickel y Smith (2004). El material fue depositado en el Herbario Metropolitano Ramón Riba y Nava Esparza (UAMIZ), de la Universidad Autónoma Metropolitana Iztapalapa. De cada ejemplar se obtuvieron esporas mediante la recolección de pinnas con esporangios maduros y cerrados, los cuales fueron colocados en sobres de papel manila y se dejaron secar durante 15 días para favorecer la liberación natural de las esporas (Pérez-García, 1988). El contenido de los sobres fue tamizado con una malla Mont Inox $\mathrm{n}^{\circ} 200$, de $0.074 \mathrm{~mm}$ de diámetro, para eliminar diversos detritos (indusio, parafisos, esporangio, tricomas, etc.). El material obtenido se guardó en viales de plástico etiquetados con el nombre de cada especie y el número de colecta, de donde se tomaron para los estudios de microscopía óptica y/o electrónica de barrido. Para el primer caso se tomaron esporas con un pincel y se colocaron sobre una gota de gelatina glicerinada en estado líquido depositada sobre un portaobjetos etiquetado con el nombre de la especie y el número de ejemplar de respaldo al que pertenece; posteriormente, se colocó un cubreobjetos y se dejó a temperatura ambiente durante 24 horas, pasado ese tiempo, se limpió el excedente y se selló utilizando barniz de uñas transparente. Las preparaciones fueron observadas al microscopio óptico (modelo Olympus BX41), en campo claro y en contraste de fases; se obtuvieron medidas de 20 esporas al azar para cada especie, relativas a los ejes polar y ecuatorial y el grosor del exosporio y del perisporio. Así mismo, se obtuvieron fotografías de las vistas usuales (polar, ecuatorial y distal) y se determinaron las características de cada espora (ornamentación, estratificación general, tipo apertural, etc.) de acuerdo con Punt et al. (2007), Kremp (1965) y Lellinger (2002). También muestras de las esporas fueron acetolizadas de acuerdo con la técnica de Erdtman (1960); todas las preparaciones elaboradas mediante esta técnica se encuentran depositadas en la palinoteca del herbario de la UAM-Iztapalapa.

Para el análisis de microscopía electrónica de barrido se tomaron esporas utilizando un pincel de pocas cerdas y se es- 
polvorearon en cinta adhesiva de doble cara conductiva de carbón, colocada sobre un portamuestras de aluminio; no se realizó la deshidratación gradual en alcoholes ni el punto crítico, pues la esporopolenina en los helechos y licofitas es más gruesa y resistente que en los granos de polen, además de que no existen evidencias de procesos harmomegáticos en ellas (E. Martínez-Hernández, com. pers.). Posteriormente se cubrieron con una capa fina de oro-paladio mediante una ionizadora Denton Vaccum Desk II y se observaron al microscopio electrónico de barrido (Jeol JSM-5310LV). Se obtuvieron imágenes digitales e impresiones en papel térmico UPP-110HD SONY (Type II, High Density), de las esporas de cada especie, incluyendo vistas polares, distales, ecuatoriales y acercamientos para observar patrones de ornamentación.

\section{Resultados}

Los ejemplares recolectados corresponden a 36 especies de helechos y tres licofitas agrupadas en 27 géneros y 14 familias de acuerdo a la clasificación de Smith et al. (2006) y Christenhusz et al. (2011). Las descripciones palinológicas incluyen el tipo de apertura, la forma en vista polar (VP) y en vista ecuatorial (VE), la coloración registrada en material fresco, medidas de los ejes (mínimo (promedio) máximo \pm desviación estándar) polar (EP) y ecuatorial (EE), el índice eje polar-eje ecuatorial (P/E), en su caso el diámetro (D), el grosor del exosporio (E), el del perisporio (Per), en su caso el tamaño de las equinas $(\mathrm{Eq})$, la ornamentación $(\mathrm{O}) \mathrm{y}$, finalmente, información del ejemplar examinado. Las imágenes de microscopía óptica (MO) se tomaron a 1000X y las de microscopía electrónica de barrido (MEB), a diversos aumentos, los cuales se indican en las respectivas microfotografías.

Descripción de las esporas.

Selaginellaceae Willk.

Selaginella pallescens (C.Presl) Spring Figura 1A, B Microspora trilete, VP: triangular a circular, VE: semicircular, Color: amarillo anaranjado, EP: 22.5 (25.9) $30 \pm 2.00$ $\mu \mathrm{m}$, EE: 26 (30.5) $32.3 \pm 1.51 \mu \mathrm{m}$, P/E. 0.84 suboblado, O: heteropolar, proximalmente gemada, distalmente clavada, las clavas de 0.98 (2.6) $3.9 \pm 1.19 \mu \mathrm{m}$. Ejemplar: FGN 279.

\section{Selaginella stenophylla A.Braun}

Figura 1C, D Microspora trilete, cingulada, VP: circular, VE: semicircular, perfil proximal cóncavo, Color: amarillo, EP: 16 (22) $29 \pm 3.17 \mu \mathrm{m}$, EE: 29 (30) $39 \pm 3.96 \mu \mathrm{m}$, P/E: 0.74 oblado, Tétrada: 39.2 (41.6) $46 \pm 2.16$, O: heteropolar, distalmente reticulada y hamuloso-rugulada con espinas en las que se observan proyecciones estrelladas, microrreticuladas y espinuladas. Ejemplares: FGN 278, FGN 280.
Equisetaceae Michx. ex D.C. Equisetum myriochaetum Schltdl. \& Cham.

Figura 1E, F Espora alete, clorofílica, con eláteres, V: esférica, Color: verde, D: 38 (46) $59 \pm 5.23 \mu \mathrm{m}$, P/E: 0.98 esférica, O: isopolar, psilada con algunos gránulos. Ejemplar: FGN 283.

\section{Dicksoniaceae M.R.Schomb. Lophosoria quadripinnata (J.F.Gmel.) C.Chr.}

Figura 1G, H

Espora trilete, VP: triangular, VE: semicircular, perfil proximal plano a punteado, Color: hialino a amarillo, EP: 63 (74) $91 \pm 7.41 \mu \mathrm{m}, \mathrm{EE}: 68$ (81) $107 \pm 10.72 \mu \mathrm{m}, \mathrm{P} / \mathrm{E}: 0.91$ oblado esferoidal, O: cingulada, heteropolar; proximalmente granulada-tuberculada, distalmente foveolada. Ejemplar: FGN 287.

\section{Dennstaedtiaceae Lotsy}

Dennstaedtia globulifera (Poir.) Hieron. Figura 2A, B Espora trilete, VP: triangular con ápice redondeado a plano, VE: reniforme a semicircular, Color: pardo claro a oscuro, EP: 21.5 (24.5) $32 \pm 2.69 \mu \mathrm{m}$, EE: 32 (36) $41 \pm 2.21 \mu \mathrm{m}$, P/E: 0.69 oblado, O: heteropolar, verrugada-papilada, con engrosamientos de 0.98 (2.5) $4.9 \pm 1.14 \mu \mathrm{m}$ en la cara distal. Ejemplar: FGN 237.

Pteridaceae E.D.M.Kirchn.

Adiantum andicola Liebm.

Figura 2C, D Espora trilete, VP: triangular, VE: globosa semicircular, Color: pardo claro, EP: 33 (35) $39 \pm 1.48 \mu \mathrm{m}$, EE: 37 (43) 48 $\pm 2.48 \mu \mathrm{m}, \mathrm{P} / \mathrm{E}: 0.80$ suboblado, O: isopolar, rugulada, con gránulos. Ejemplares: FGN 264, FGN 277.

\section{Cheilanthes notholaenoides (Desv.) Maxon ex Weath.}

Figura 2E, F

Espora trilete con perisporio evidente al microscopio óptico, VP: subtriangular, triangular convexa a circular, VE: circular, Color: pardo claro, EP: 43 (47) $50 \pm 1.92 \mu \mathrm{m}$, EE: 46 (51.5) $57 \pm 2.52 \mu \mathrm{m}$, P/E: 0.90 oblado esferoidal, Per: 0.98 (2) $2.94 \pm 0.62 \mu \mathrm{m}$, columnelada, O: isopolar, fosulada con gránulos (MEB); patrón microreticulado (MO). Ejemplar: FGN 253.

\section{Llavea cordifolia Lag.}

Figura 2G, $\mathrm{H}$ Espora trilete, VP: subtriangular a triangular, VE: semicircular, perfil proximal plano a punteado, Color: pardo anaranjado, EP: 27.5 (29) $32 \pm 1.47 \mu \mathrm{m}$, EE: 32 (37) $40 \pm 2.04 \mu \mathrm{m}$, P/E: 0.79 suboblado, L: 15.6 (18) $22.5 \pm 1.64 \mu \mathrm{m}$, O: isopolar, gemada. Ejemplar: FGN 248.

Mildella intramarginalis (Link) Trevis. Figura 3A, B Espora trilete con perisporio evidente al microscopio óptico, VP: circular, VE: circular a semicircular, Color: pardo oscuro, EP: 56 (62.5) $66 \pm 2.84 \mu \mathrm{m}$, EE: 58 (65) $69.5 \pm 3.4 \mu \mathrm{m}$, 
A

C

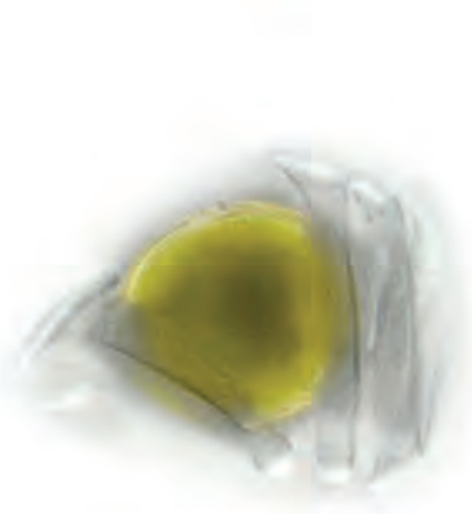

$\mathrm{E}$

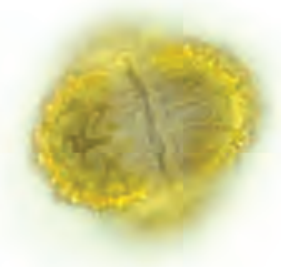

G

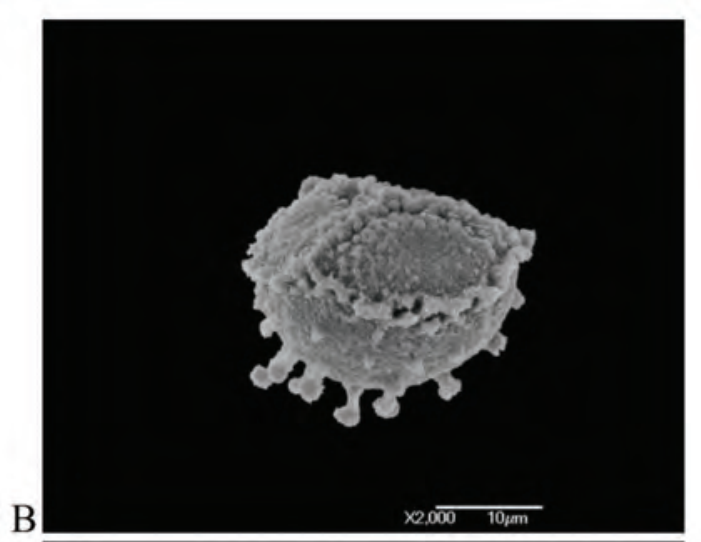

$\mathrm{B}$
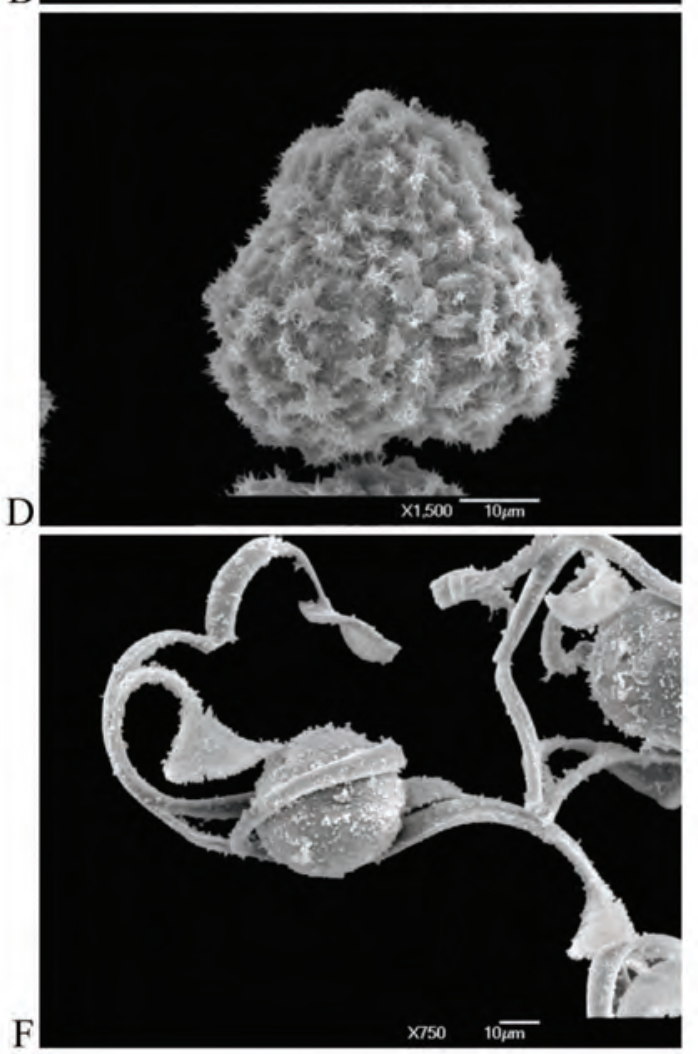

$\mathrm{F}$

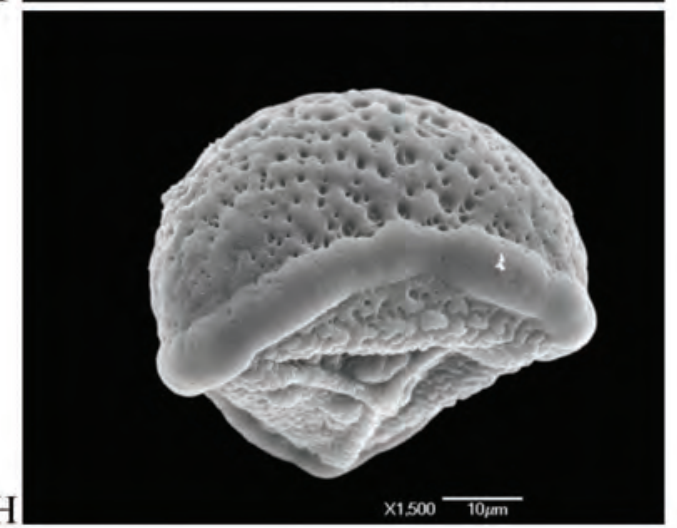

Figura 1. Selaginellaceae (microesporas): Selaginella pallescens, A: vista ecuatorial, MO, B: vista ecuatorial, MEB; S. stenophylla, C: tétrada, MO, D: tétrada, MEB; Equisetaceae: Equisetum myriochaetum, E: mónada, MO, F: mónadas, MEB; Lophosoriaceae: Lophosoria quadripinnata, G: vista distal, corte óptico y análisis L-O, MO, H: vista ecuatorial, MEB. 

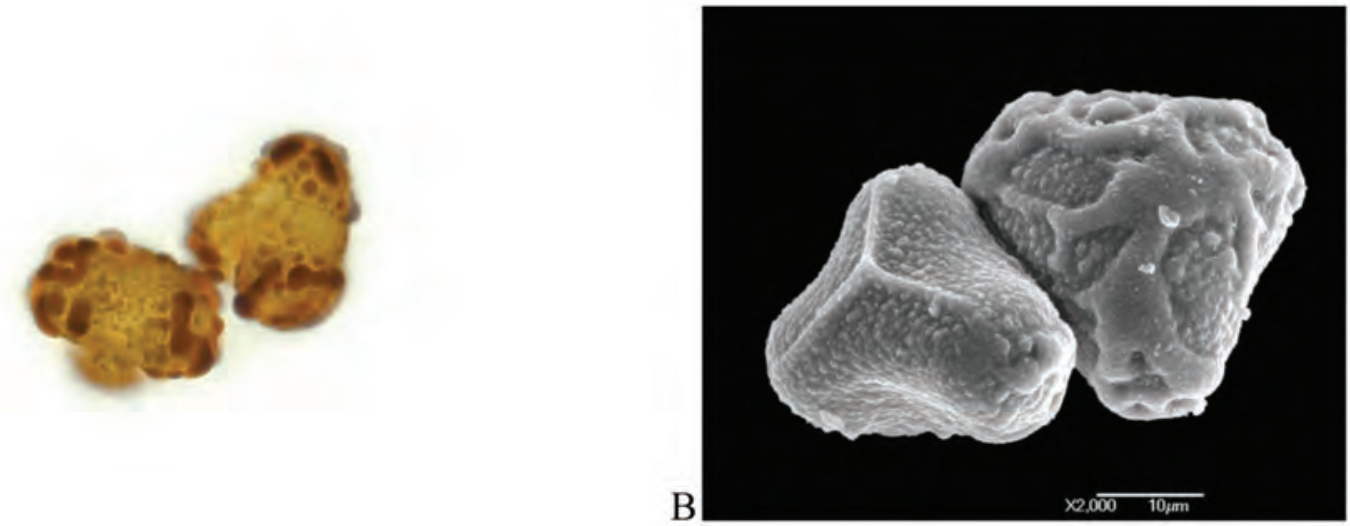

A

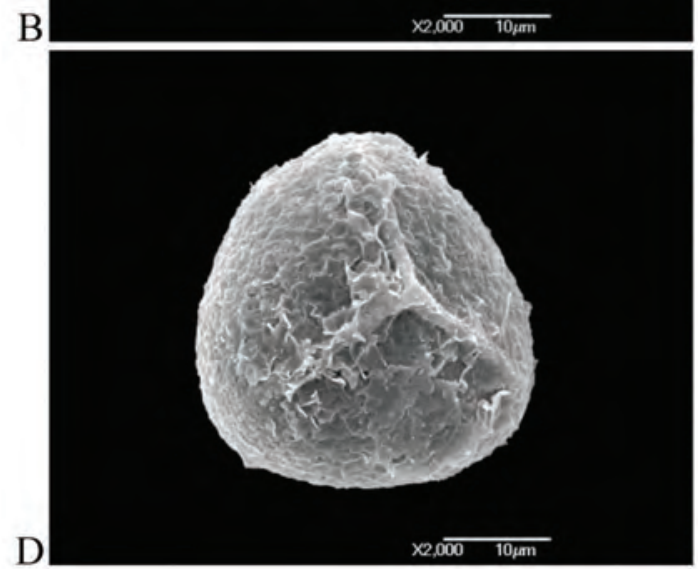

C

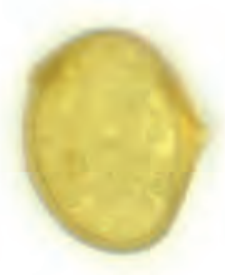

$\mathrm{D}$

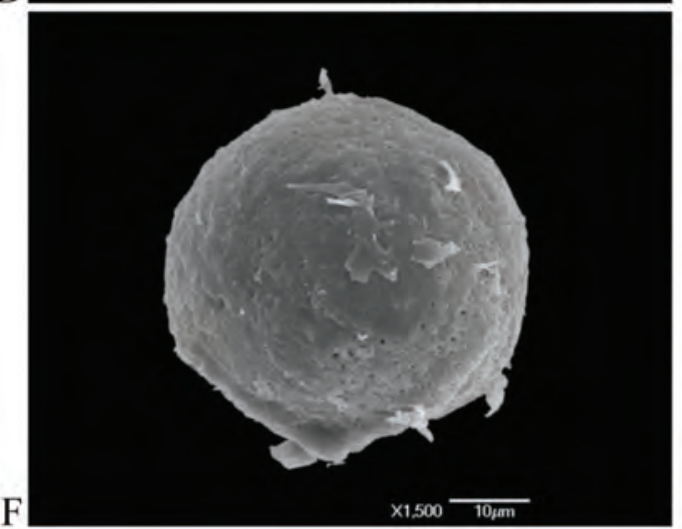

E
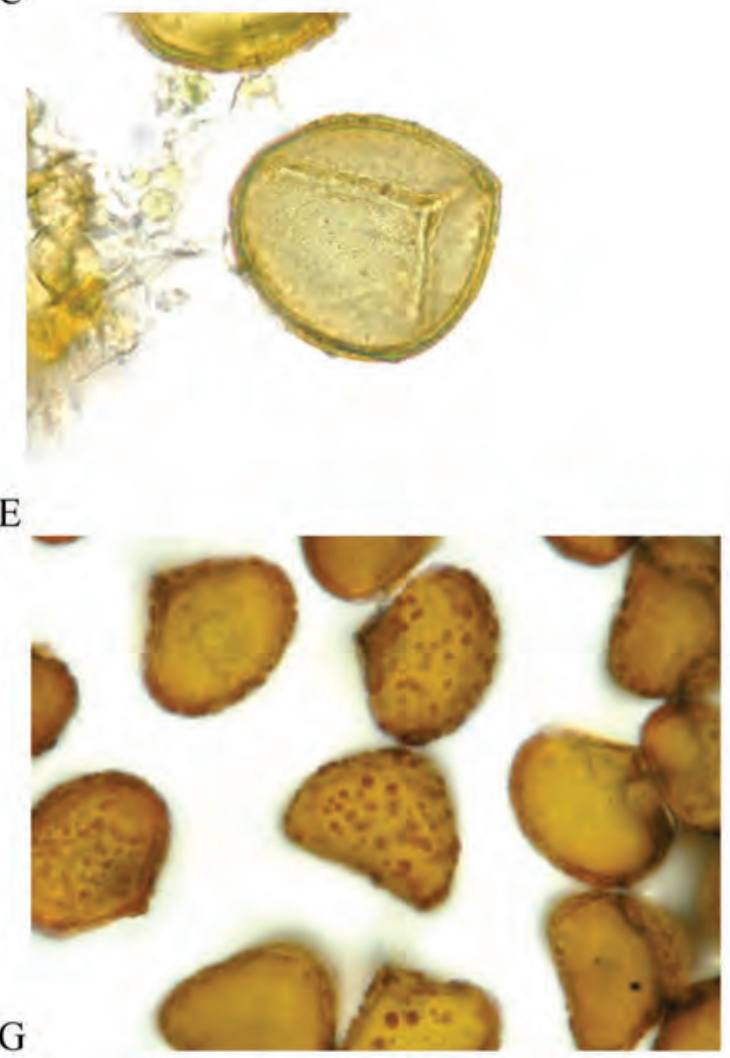

$\mathrm{F}$

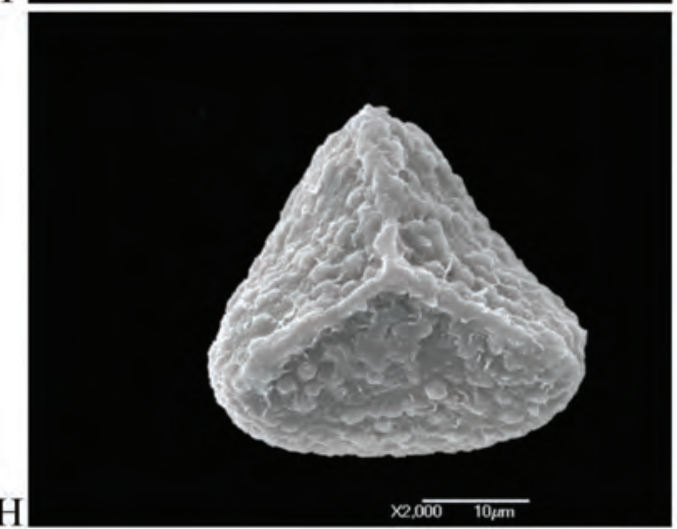

Figura 2. Dennstaedtiaceae: Dennstaedtia globulifera, A: vista ecuatorial, MO, B: vista proximal y distal, MEB; Pteridaceae: Adiantum andicola, C: vista ecuatorial, MO, D: vista proximal, MEB; Cheilanthes notholaenoides, E: vista proximal, MO, F: vista ecuatorial, MEB; Llavea cordifolia, G: varias vistas, MO, H: vista proximal, MEB. 


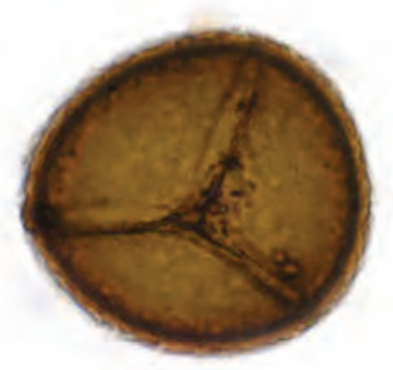

A
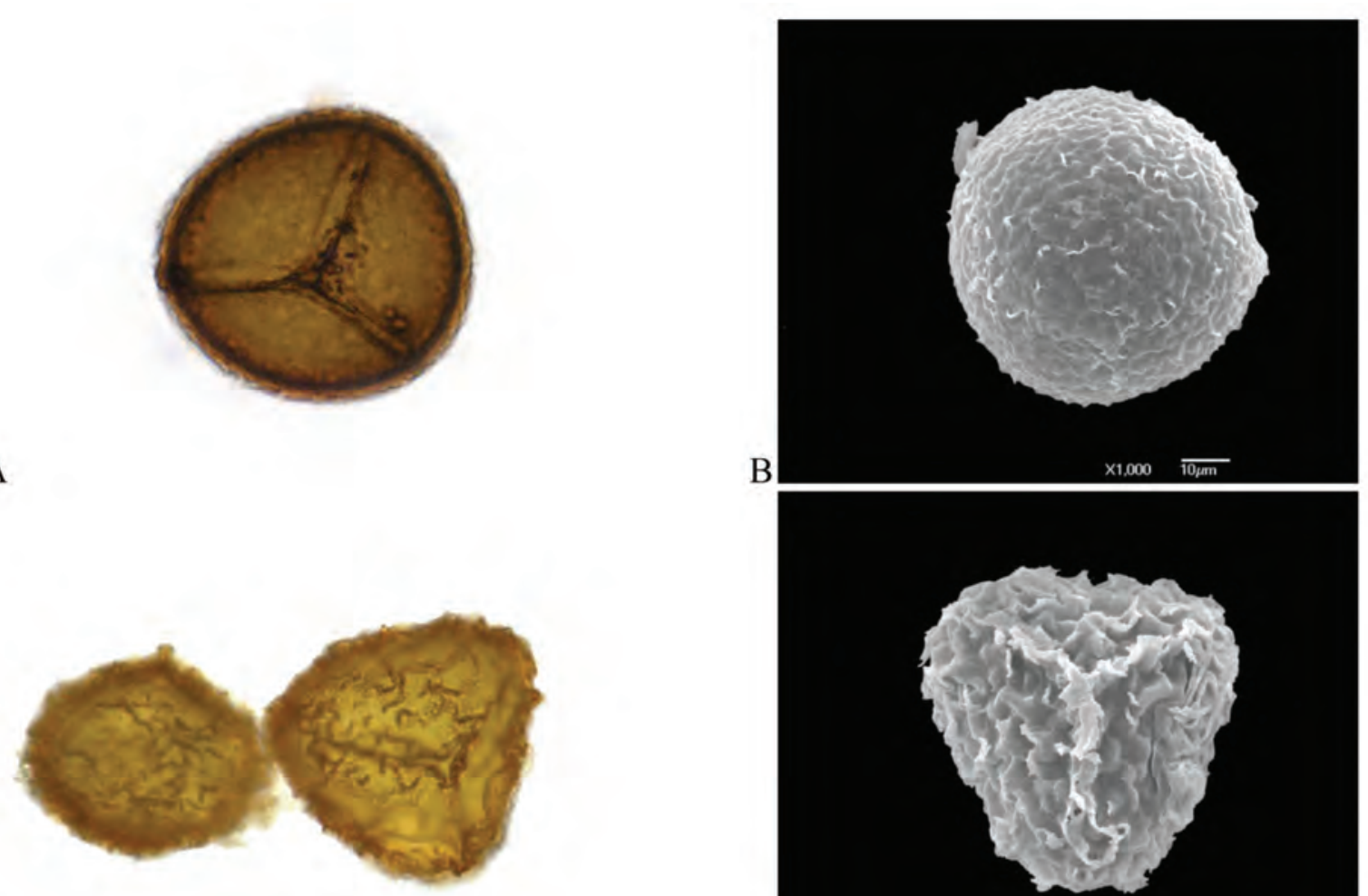

C
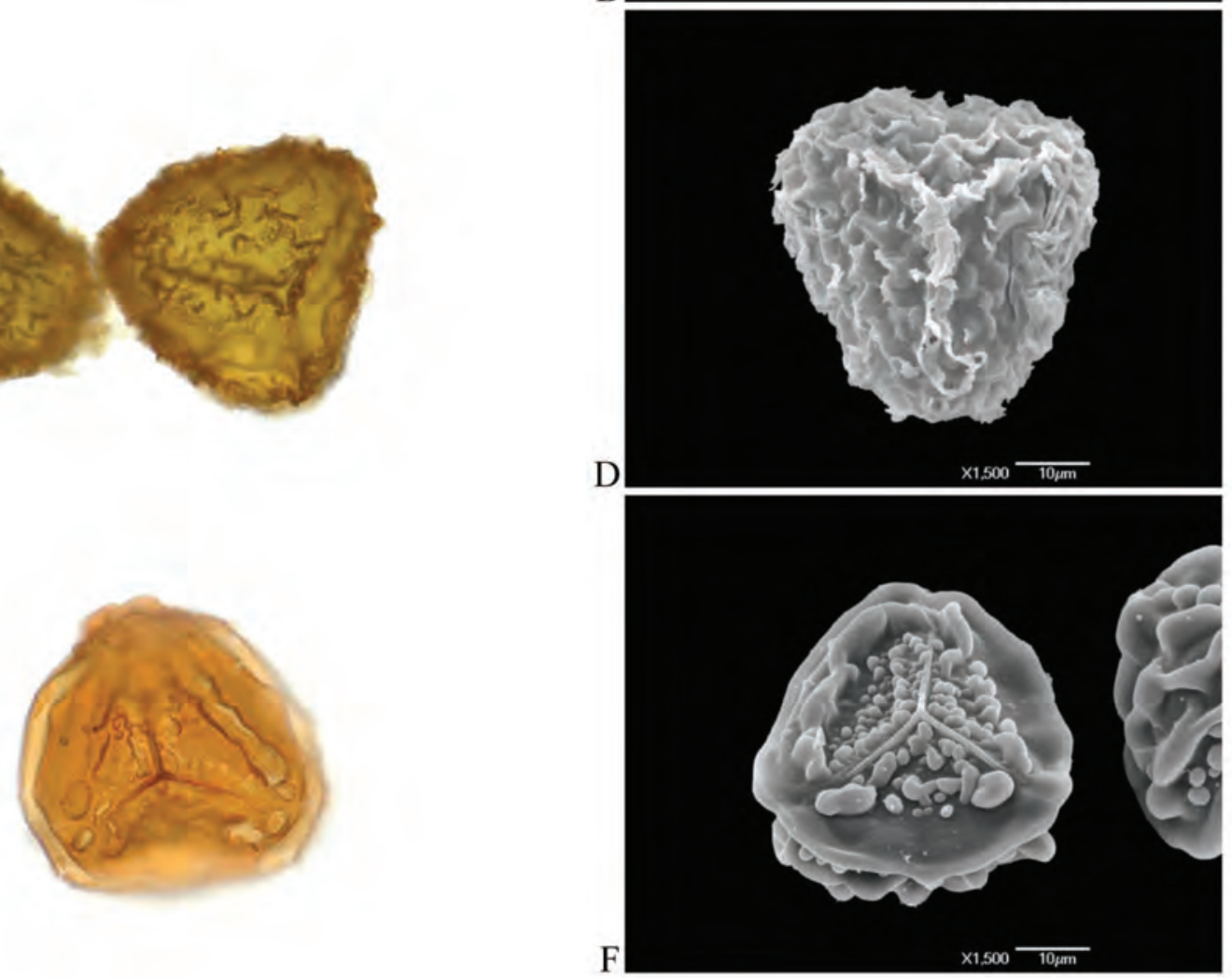

E

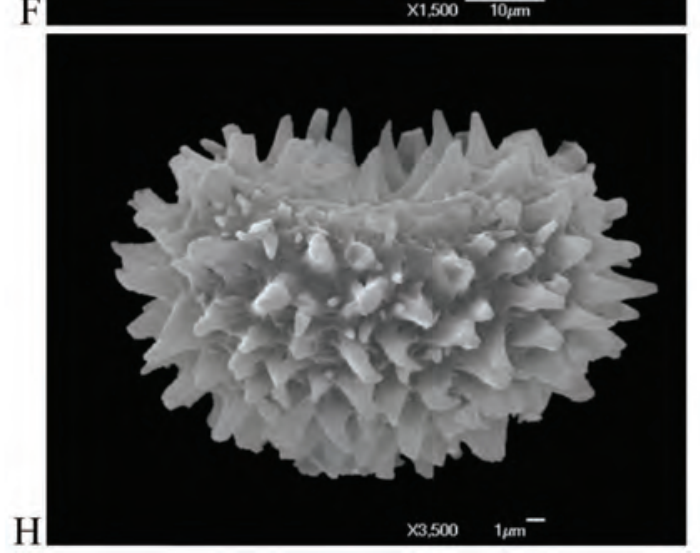

G

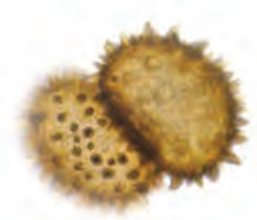

G

$\mathrm{H}$

Figura 3. Pteridaceae (cont.): Mildella intramarginalis, A: vista proximal, MO, B: vista ecuatorial, MEB; Pellaea ovata, C: vista ecuatorial y proximal, MO, D: vista proximal, MEB; Pteris quadriaurita, E: vista proximal, MO, F: vista proximal, MEB; Woodsiaceae: Cystopteris fragilis, G: vista distal y ecuatorial, MO, H: vista ecuatorial, MEB. 
P/E: 0.96 oblado esferoidal, Ex: 0.98 (1) $1.96 \pm 0.2 \mu \mathrm{m}$, Per: $1.96(2.8) 3.92 \pm 0.62 \mu \mathrm{m}, \mathrm{O}$ : isopolar, rugulada-crestada. Ejemplar: FGN 226.

Pellaea ovata (Desv.) Weath.

Figura 3C, D Espora trilete, VP: triangular-convexa, VE: semicircular, Color: pardo, EP: 37 (45) $54 \pm 3.68 \mu \mathrm{m}$, EE: 40 (51.5) $60 \pm$ $5.75 \mu \mathrm{m}, \mathrm{P} / \mathrm{E}: 0.88$ oblado esferoidal, $\mathrm{O}$ : isopolar, rugulada con crestas, más numerosas en la cara distal que en la proximal. Ejemplar: FGN 260.

\section{Pteris quadriaurita Retz.}

Figura 3E, F

Espora trilete con perisporio evidente al MO, VP: triangular-circular, VE: semicircular, perfil proximal plano, Color: anaranjado a rojizo, EP: 37 (45) $56 \pm 5.33 \mu \mathrm{m}$, EE: 46 (57) $70 \pm 7.33 \mu \mathrm{m}$, P/E: 0.79 suboblado, D: 48 (58) $62.7 \pm 5.33$ $\mu \mathrm{m}$, O: cingulada, heteropolar; proximalmente granuladogemulada, distalmente rugulado-hamulosa. Ejemplar: FGN 289.

\section{Woodsiaceae Herter}

Cystopteris fragilis (L.) Bernh.

Figura 3G, H

Espora monolete, VP: elipsoidal, VE: reniforme, Color: pardo obscuro, EP: 21.5 (26) $30 \pm 2.43 \mu \mathrm{m}$, EE: 30 (34.5) $39 \pm 2.41 \mu \mathrm{m}, \mathrm{P} / \mathrm{E}: 0.68$ oblado, Eq: 3 (4) $7 \pm 0.95 \mu \mathrm{m}, \mathrm{O}:$ isopolar, equinada, espinas foveoladas en la base. Ejemplar: FGN 242.

\section{Diplazium lonchophyllum Kunze}

Figura 4A, B Espora monolete con perisporio muy evidente al microscopio óptico, VP: elipsoidal, VE: elipsoidal-reniforme, Color: pardo claro, EP: 31 (36) $43 \pm 3.51 \mu \mathrm{m}$, EE: 34 (45) $54 \pm$ $4.23 \mu \mathrm{m}, \mathrm{P} / \mathrm{E}$ : 0.81 suboblado, O: isopolar, plegado, pliegues romos fusionados. Ejemplar: FGN 233.

\section{Aspleniaceae Newman}

Asplenium abscissum Willd. Figura 4C, D Espora monolete con perisporio muy evidente al microscopio óptico, VP: elipsoidal, VE: reniforme, Color: pardo, EP: 21 (27) $31 \pm 2.4 \mu \mathrm{m}$, EE: 34 (38) $43 \pm 2.61 \mu \mathrm{m}, \mathrm{P} / \mathrm{E}: 0.74$ oblado, Per: 3 (5) $7 \mu \mathrm{m}$, O: isopolar, alas crestadas, parcialmente fusionadas, a veces foveoladas en la base, espinas entre las alas. Ejemplar: FGN 224.

\section{Asplenium monanthes L.}

Figura 4E, F Espora monolete con perisporio evidente al microscopio óptico, VP: elipsoidal, VE: reniforme, Color: pardo, EP: 39 (45) $51 \pm 3.57 \mu \mathrm{m}$, EE: 53 (60) $71 \pm 4.9 \mu \mathrm{m}, \mathrm{P} / \mathrm{E}: 0.74$ oblado, Per: 2 (6.5) $10 \pm 2.5 \mu \mathrm{m}$, O: isopolar, perisporio alado-fenestrado, alas parcialmente fusionadas con espínulas. Ejemplar: FGN 222.

\section{Asplenium sessilifolium Desv. var. sessilifolium}

Figura 4G, H

Espora monolete con perisporio evidente al microscopio óptico, VP: elipsoidal, VE: reniforme, Color: pardo, EP: 23.5 (29) $38 \pm 3.19 \mu \mathrm{m}$, EE: 31 (38) $44 \pm 2.36 \mu \mathrm{m}, \mathrm{P} / \mathrm{E}: 0.78$ suboblado, Per: 4 (5.5) $9 \pm 1.34 \mu \mathrm{m}$, O: isopolar, perisporio alado, las alas parcialmente fusionadas formando un patrón reticulado, con espinas en margen de alas y entre ellas. Ejemplar: FGN 259.

\section{Holodictyum ghiesbreightii (E.Fourn.) Maxon}

Figura 5A, B

Espora monolete con perisporio evidente al microscopio óptico, VP: elipsoidal (trapezoidal con el perisporio), VE: reniforme (penta o hexagonal, con el perisporio), Color: pardo, EP: 36 (44) $50 \pm 4.5 \mu \mathrm{m}$, EE: 53 (59) $70 \pm 5.22 \mu \mathrm{m}$, P/E: 0.74 oblado, Per: 3.92 (8.5) $14.7 \pm 2.6 \mu \mathrm{m}$, O: isopolar, reticulada, alada. Ejemplar: FGN 219.

\section{Thelypteridaceae Pic.Serm. Macrothelypteris torresiana (Gaudich.) Ching}

Figura 5C, D Espora monolete, VP: elipsoidal, VE: reniforme, Color: amarillo-claro, EP: 29 (37) $50 \pm 4.61 \mu \mathrm{m}$, EE: 35 (51) $60 \pm 5.35 \mu \mathrm{m}$, P/E: 0.72 oblado, O: isopolar, hamulosoreticulado con crestas equinadas, las espinas de 2.94 (4.5) $7.84 \pm 1.07 \mu \mathrm{m}$, a veces foveoladas en su base. Ejemplar: FGN 284.

Thelypteris ovata R.P.St.John var. lindheimeri (C.Chr.) A.R.Sm. Figura 5E, F Espora monolete con perisporio evidente al microscopio óptico, VP: elipsoidal, VE: reniforme-pentagonal, Color: pardo oscuro, EP: 25 (30) $34 \pm 2.59 \mu \mathrm{m}$, EE: 38 (44) $51 \pm 3.37 \mu \mathrm{m}, \mathrm{P} / \mathrm{E}: 0.67$ oblado, Per: 3 (5) $6.8 \pm 0.97 \mu \mathrm{m}, \mathrm{O}$ : isopolar, lofada, perisporio con pliegues romos foveolados y fusionados delimitando áreas ruguladas con perforaciones. Ejemplar: FGN 251.

\section{Blechnaceae Newman \\ Blechnum appendiculatum Willd.}

Figura 5G, $\mathrm{H}$ Espora monolete con perisporio evidente al microscopio óptico, VP: elipsoidal, VE: reniforme, Color: pardo claro, EP: 22.5 (28) $31 \pm 2.72 \mu \mathrm{m}, \mathrm{EE}: 34$ (40) $45 \pm 2.87 \mu \mathrm{m}, \mathrm{P} / \mathrm{E}:$ 0.695 oblado, O: isopolar, rugulada, microreticulada, con escasos gránulos. Ejemplar: FGN 252.

Woodwardia martinezii Maxon ex Weath. Figura 6A, B Espora monolete con perisporio evidente al microscopio óptico, VP: elipsoidal, VE: reniforme, Color: amarillo claro, EP: 29 (54) $66 \pm 9.96 \mu \mathrm{m}$, EE: 60 (78) $90 \pm 9.4 \mu \mathrm{m}$, P/E. 0.67 oblado, Ex: 2 (3) $4 \pm 0.78 \mu \mathrm{m}$, Per: 2.94 (6) $19 \pm 3.74 \mu \mathrm{m}$, $\mathrm{O}$ : isopolar, perisporio plegado-crestado, rugulado, crestas parcialmente fusionadas. Ejemplar: FGN 288. 


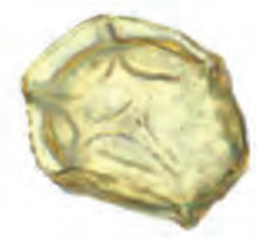

A

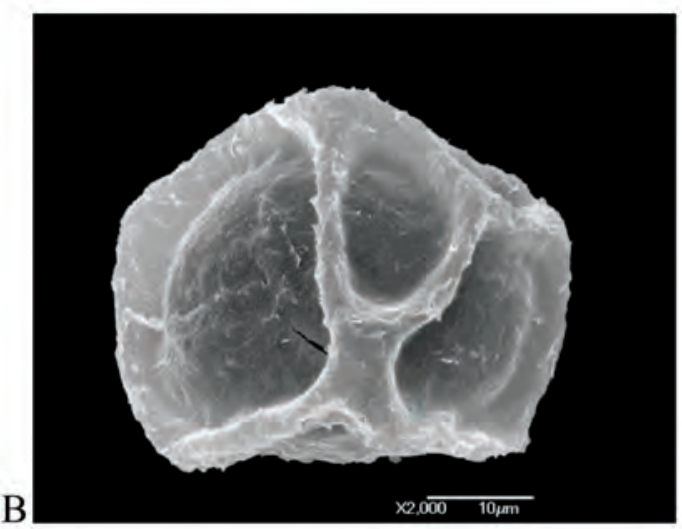

C
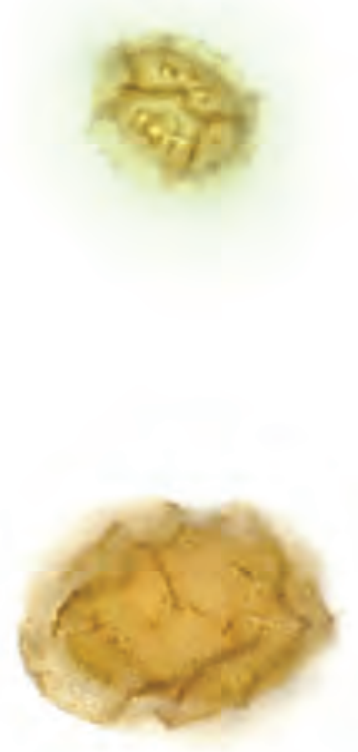

D

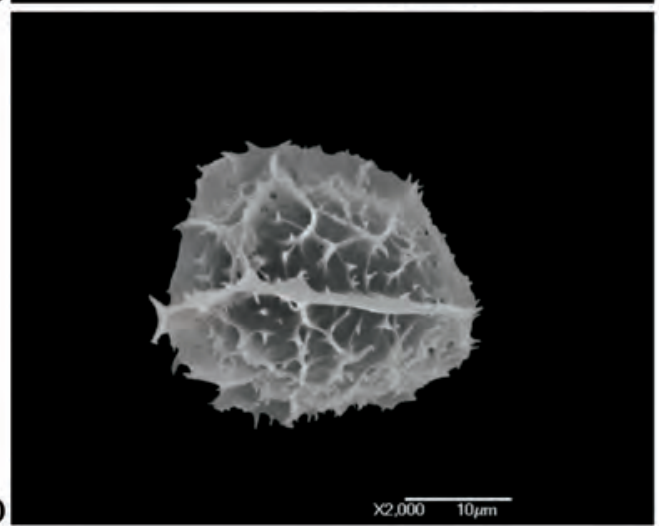

E
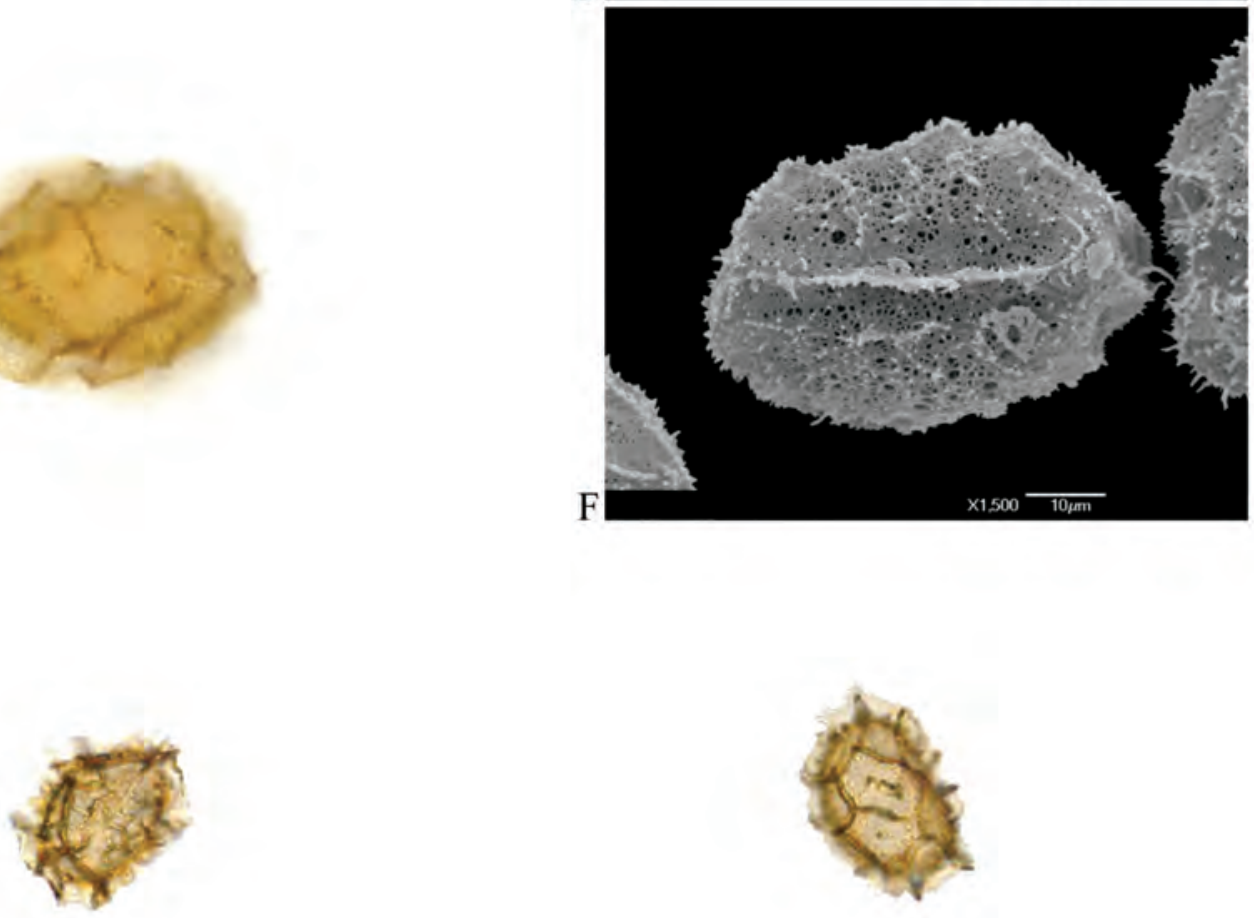

G

$\mathrm{H}$

Figura 4. Woodsiaceae (cont.): Diplazium lonchophyllum, A: vista ecuatorial, MO, B: vista ecuatorial, MEB; Aspleniaceae: Asplenium abscissum, C: vista distal, MO, D: vista proximal, MEB; A. monanthes, E: vista distal, MO, F: vista proximal MEB; A. sessilifolium var. sessilifolium, G: vista proximal, $\mathrm{MO}, \mathrm{H}$ : vista distal, MO. 


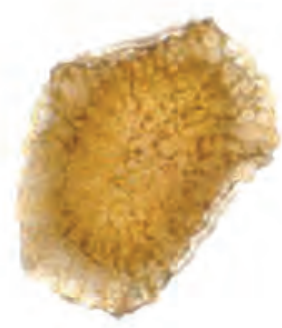

A

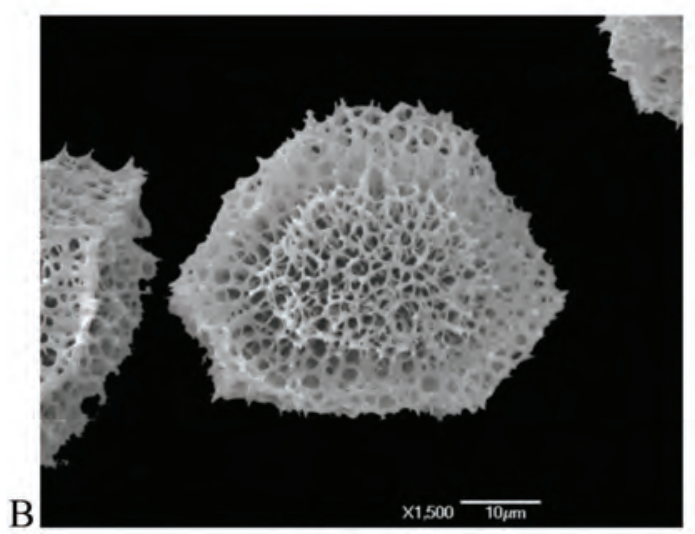

$\mathrm{C}$
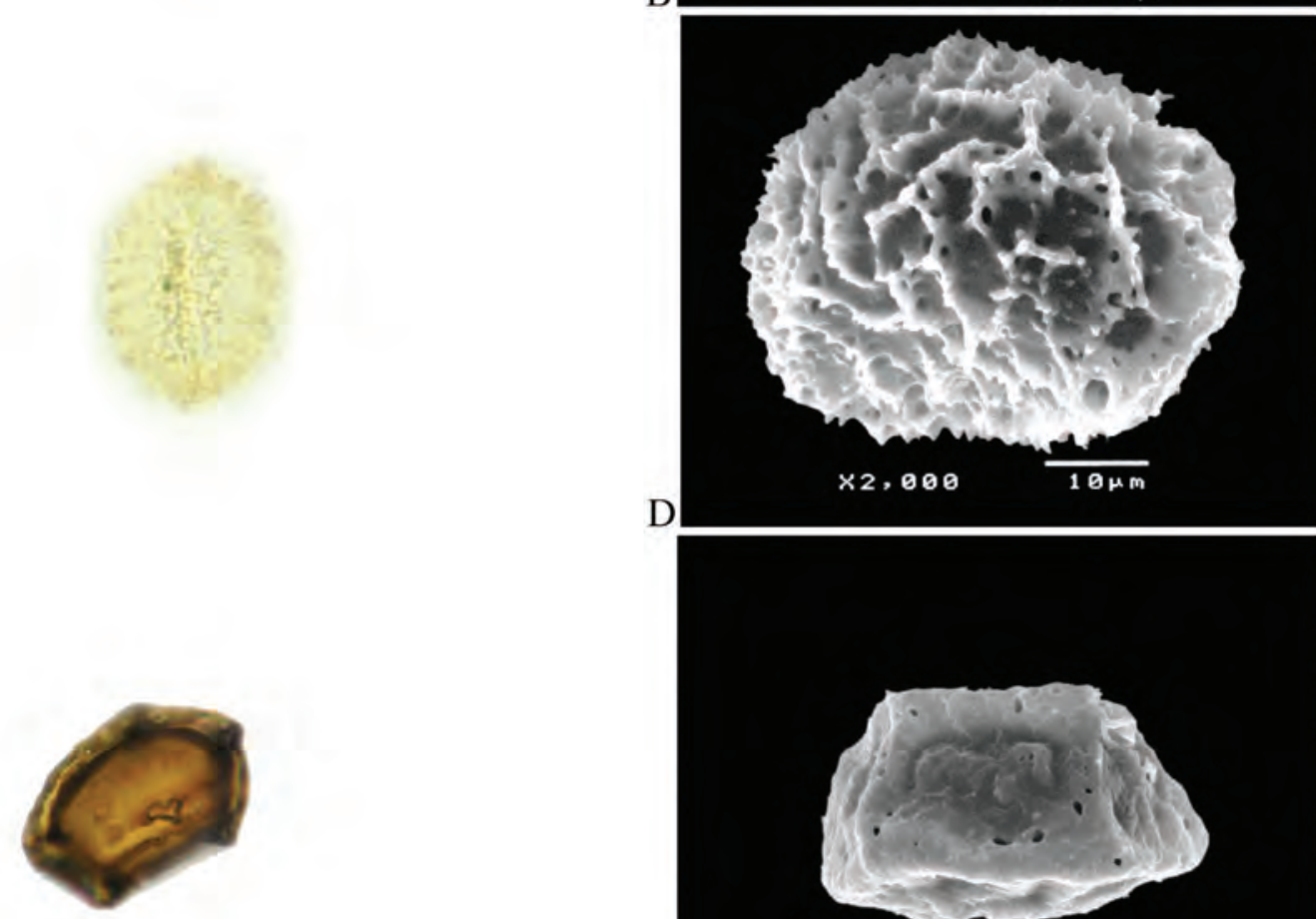

$\mathrm{D}$

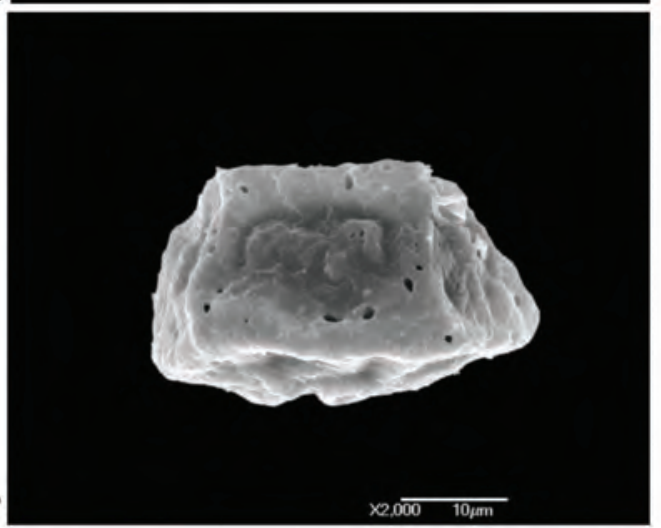

E

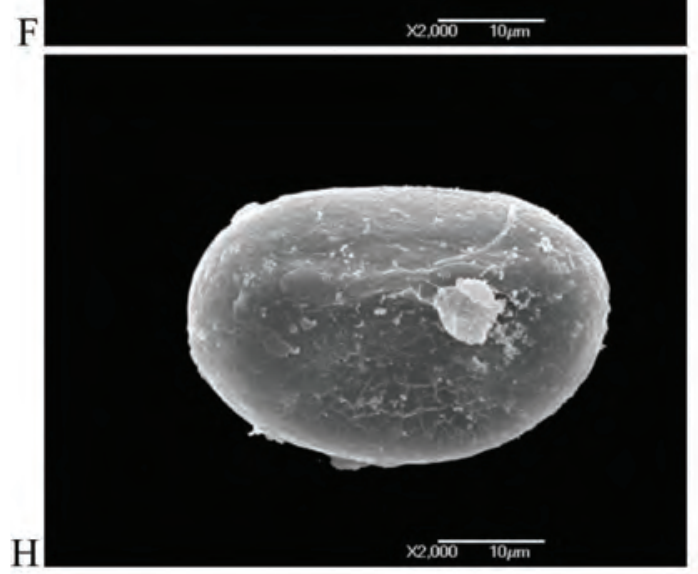

G

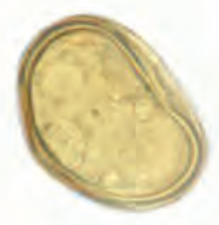

Figura 5. Aspleniaceae (cont.): Holodyctium ghiesbreghtii, A: vista ecuatorial, MO, B: vista ecuatorial, MEB; Thelypteridaceae: Macrothelypteris torresiana, C: vista proximal, MO, D: vista ecuatorial, MEB; Thelypteris ovata var. lindheimeri, E: vista ecuatorial, MO, F: vista ecuatorial, MEB; Blechnaceae: Blechnum appendiculatum, G: vista ecuatorial, MO, H: vista proximal, MEB. 


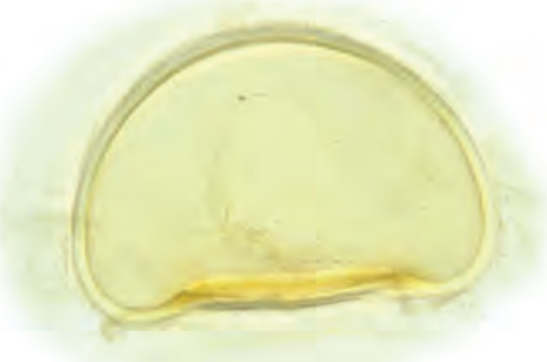

A

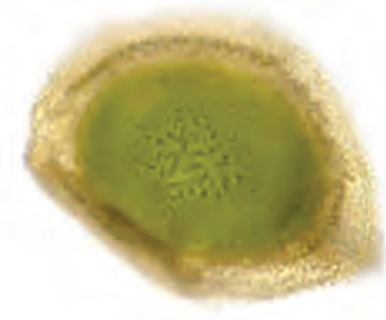

$\mathrm{C}$

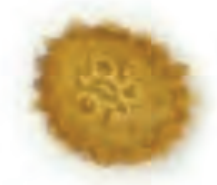

E

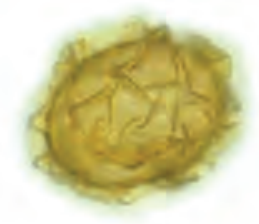

G
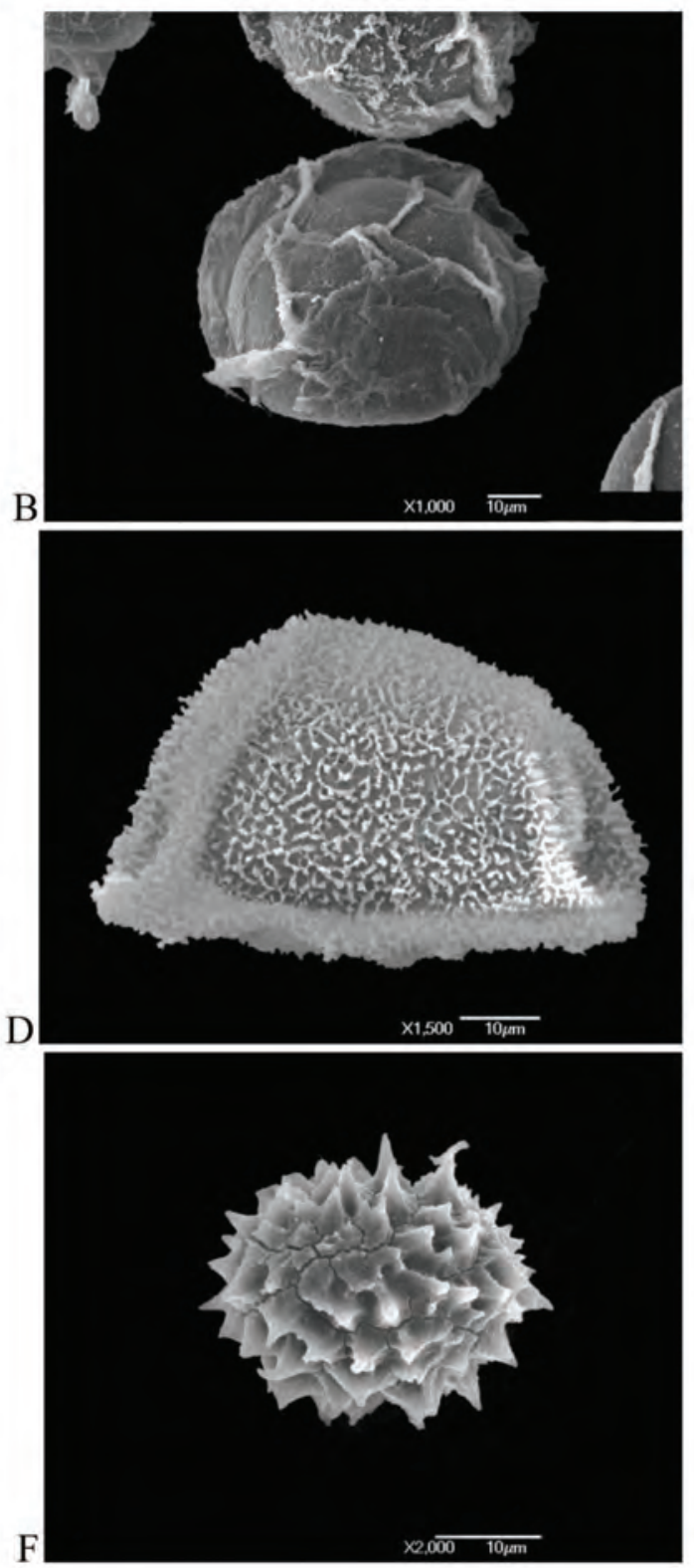

$\mathrm{F}$

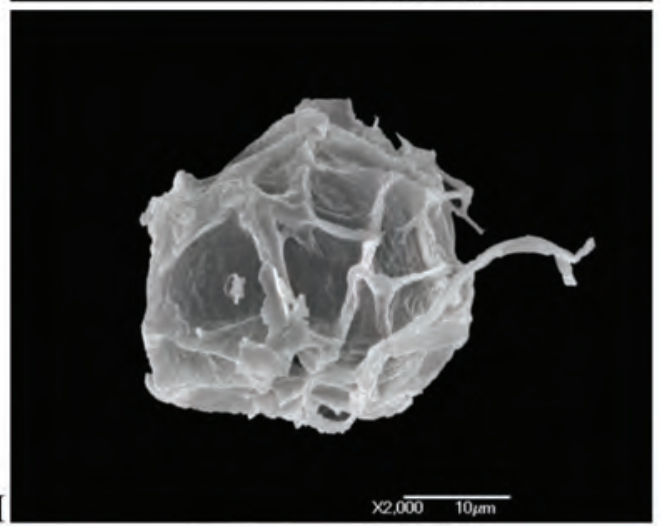

Figura 6. Blechnaceae (cont.): Woodwardia martinezii, A: vista ecuatorial, MO, B: vista ecuatorial, MEB; Onocleaceae: Onocleopsis hintonii, C: vista ecuatorial, MO, D: vista ecuatorial, MEB; Dryopteridaceae: Ctenitis equestris, E: vista distal, MO, F: vista distal, MEB; Elaphoglossum obscurum, G: vista distal, MO, H: vista ecuatorial, MEB. 
Onocleaceae Pic.Serm.

Onocleopsis hintonii F.Ballard Figura 6C, D

Espora monolete, clorofílica, con perisporio evidente al microscopio óptico, VP: elipsoidal, VE: elipsoidal a reniforme, Color: verde, EP: 40 (48) $60 \pm 5.36 \mu \mathrm{m}$, EE: 56 (66) 76 $\pm 4.52 \mu \mathrm{m}$, P/E: 0.73 oblado, O: isopolar, microreticulada, con crestas y espínulas. Ejemplar: FGN 282

\section{Dryopteridaceae Herter \\ Ctenitis equestris (Kunze) Ching var. equestris}

Figura 6E, F

Espora monolete, VP: elipsoidal-circular, VE: elipsoidal, Color: pardo, EP: 19 (24) $29 \pm 2.03 \mu$ m, EE: 24 (31) $39 \pm$ $3.5 \mu \mathrm{m}, \mathrm{P} / \mathrm{E}: 0.78$ suboblado, Eq: 2.9 (6.1) $9.8 \pm 2.26 \mu \mathrm{m}$, $\mathrm{O}$ : isopolar, equinada, espinas escasamente foveoladas en su parte media. Ejemplar: FGN 238.

\section{Elaphoglossum obscurum (E.Fourn) C.Chr.}

Figura $6 \mathrm{G}, \mathrm{H}$

Espora monolete con perisporio evidente al microscopio óptico, VP: elipsoidal, VE: reniforme, Color: pardo claro, EP: 26.5 (34) $38 \pm 2.54 \mu \mathrm{m}, \mathrm{EE}: 36$ (43) $48 \pm 2.99 \mu \mathrm{m}, \mathrm{P} / \mathrm{E}$ : 0.79 suboblado, Per: 2 (4) $7 \pm 1.4 \mu \mathrm{m}$, O: isopolar, perisporio rugulado, formando pliegues de apariencia reticular. Ejemplar: FGN 257.

\section{Phanerophlebia remotispora (Schtdl. \& Cham.) C.Presl}

Figura 7A, B

Espora monolete con perisporio evidente al microscopio óptico, VP: elipsoidal, VE: reniforme, Color: pardo claro, EP: 20.5 (30.5) $35 \pm 3.34 \mu \mathrm{m}$, EE: 35 (40) $44 \pm 2.79 \mu \mathrm{m}$, P/E: 0.76 suboblada, O: isopolar, perisporio con numerosos pliegues gruesos y superficie vermiculada-reticulada. Ejemplar: FGN 227.

\section{Polystichum turrialbae Christ.}

Figura 7C, D

Espora monolete con perisporio evidente al microscopio óptico, VP: elipsoidal, VE: reniforme, Color: pardo, EP: 35 (44) $52 \pm 3.46 \mu \mathrm{m}$, EE: 45 (52) $61 \pm 0.69 \mu \mathrm{m}, \mathrm{P} / \mathrm{E}: 0.65$ oblado, Per: 3.92 (7.3) $10.7 \pm 1.83 \mu \mathrm{m}$, O: isopolar, perisporio reticulado, con crestas equinadas. Ejemplar: FGN 254.

\section{Tectariaceae Panigrahi}

Tectaria heracleifolia (Willd.) Underw. $\quad$ Figura 7E, F Espora monolete con perisporio evidente al microscopio óptico, VP: elipsoidales, VE: reniforme, Color: pardo claro, EP: 24 (33) $40 \pm 2.98 \mu \mathrm{m}$, EE: 36 (45) $51 \pm 4.01 \mu \mathrm{m}$, P/E. 0.73 oblado, Per: 1.96 (5.5) $10.7 \pm 2.15 \mu \mathrm{m}$, O: isopolar, perisporio plegado foveolado-equinulado. Ejemplar: FGN 235.

Polypodiaceae J.Presl \& C.Presl

Campyloneurum angustifolium (Sw.) Fée Figura 7G, H Espora monolete, VP: elipsoidal, VE: reniforme, Color: traslúcido a amarillo claro, EP: 30 (34) $36 \pm 1.96 \mu \mathrm{m}$, EE: 56 (58.5) $62 \pm 1.92 \mu \mathrm{m}$, P/E: 0.57 oblado, E: 2 (2.5) $4 \pm 0.56 \mu \mathrm{m}, \mathrm{O}$ : isopolar, verrugada, evidente en campo claro (MO). Ejemplar: FGN 271.

Campyloneurum xalapense Fée

Figura 8A, B Espora monolete, VP: elipsoidal, VE: reniforme, Color: traslúcido a amarillo claro, EP: 19 (32) $40 \pm 4.56 \mu \mathrm{m}$, EE: 38 (53) $63 \pm 6.73 \mu \mathrm{m}, \mathrm{P} / \mathrm{E}: 0.61$ oblado, E: 0.98 (1.5) $1.96 \pm 0.50 \mu \mathrm{m}, \mathrm{O}$ : isopolar, verrugada. Ejemplares: FGN 244, FGN 295.

\section{Pecluma sursumcurrens (Copel.) M.G.Price}

Figura 8C, D Espora monolete, VP: elipsoidal, VE: plano-convexa, Color: amarillo, EP: 35 (39) $44 \pm 2.25 \mu \mathrm{m}$, EE: 50 (54.5) $62 \pm 2.84 \mu \mathrm{m}$, Ex: 3 (4) $5 \pm 0.59 \mu \mathrm{m}$, P/E: 0.70 oblado, O: isopolar, verrugada, las verrugas de apariencia hexagonal, más grandes en la cara distal. Ejemplares: FGN 220, FGN 247.

Pleopeltis crassinervata (Fée) T.Moore Figura 8E, F Espora monolete, VP: elipsoidal, VE: reniforme, Color: amarillo, EP: 38 (41) $44 \pm 1.91 \mu \mathrm{m}$, EE: 55 (59) $63 \pm 2.56 \mu \mathrm{m}, \mathrm{P} /$ E: 0.69 oblado, Ex: 0.98 (1.13) $1.96 \pm 0.35 \mu \mathrm{m}$, O: isopolar, verrugada. Ejemplar: FGN 241.

Polypodium furfuraceum Schltdl. \& Cham. Figura 8G, H Espora monolete, VP: elipsoidal, VE: reniforme, Color: amarillo, EP: 36 (40) $44 \pm 2.41 \mu \mathrm{m}$, EE: 48 (56) $61 \pm 3.48 \mu \mathrm{m}$, P/E: 0.71 oblado, O: isopolar, verrugada (poco evidente al MO), con escasos glóbulos. Ejemplar: NVP 2.

Polypodium lepidotrichum (Fée) Maxon Figura 9A, B Espora monolete, VP: elipsoidal, VE: reniforme, Color: amarillo, EP: 28 (32) $37 \pm 2.17 \mu \mathrm{m}$, EE: 41 (49.5) $53 \pm 3.15 \mu \mathrm{m}$, P/E: 0.65 oblado, Ex: 1.96 (2.7) $2.94 \pm 0.40 \mu \mathrm{m}$, O: isopolar, vermiculada, con numerosos glóbulos, éstos de 2.9 (3.5) 4.9 $\pm 0.80 \mu \mathrm{m}$. Ejemplar: AMR 1443 .

Polypodium longepinnulatum E.Fourn. Figura 9C, D Espora monolete, VP: elipsoidal, VE: reniforme, Color: amarillo, EP: 23.5 (26) $29 \pm 1.55 \mu \mathrm{m}$, EE: 39 (43.5) $51 \pm 3.15 \mu \mathrm{m}, \mathrm{P} / \mathrm{E}: 0.60$ oblado, Ex: 0.98 (1.71) $1.96 \pm 0.43$ $\mu \mathrm{m}$, O: isopolar, verrugada, las verrugas más grandes en la cara distal, de 1.96 (3.7) $5.8 \pm 0.98 \mu \mathrm{m}$. Ejemplar: NVP 8.

Polypodium plebeium Schltdl. \& Cham. Figura 9E, F Espora monolete, VP: elipsoidal, VE: reniforme, Color: amarilla, EP: 27 (30) $33 \pm 2.29 \mu \mathrm{m}$, EE: 39 (43) $48 \pm 3.13 \mu \mathrm{m}$, P/E. 0.70 oblado, Ex: 2 (2.3) $2.9 \pm 0.49 \mu \mathrm{m}$, O: isopolar, verrugada. Ejemplar: FGN 285.

Polypodium plesiosorum Kunze Figura 9G, H Espora monolete, VP: elipsoidal, VE: reniforme, Color: 


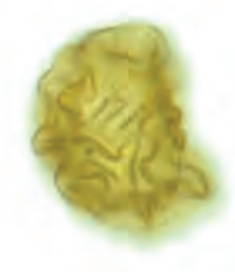

A

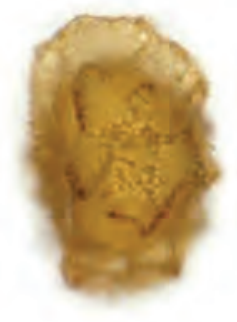

C

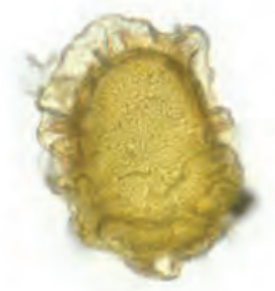

E

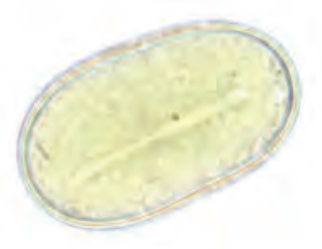

G

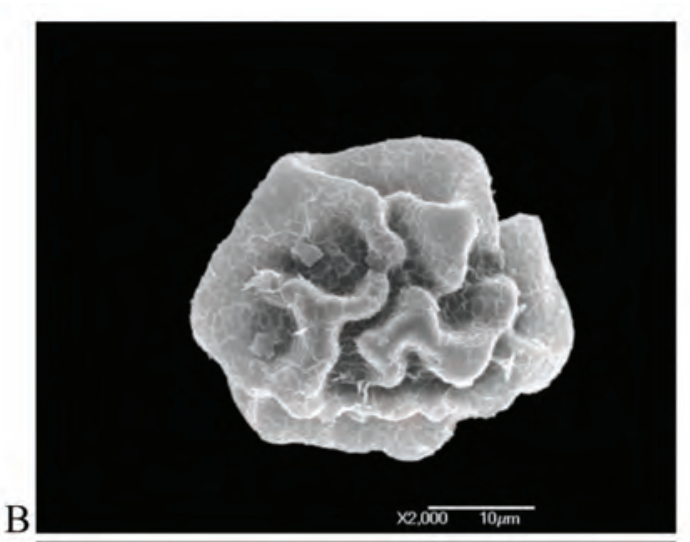

$\mathrm{B}$

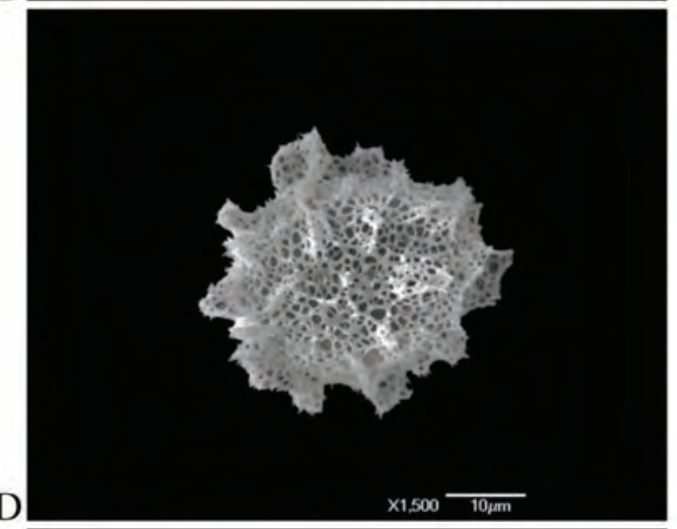

$\mathrm{D}$

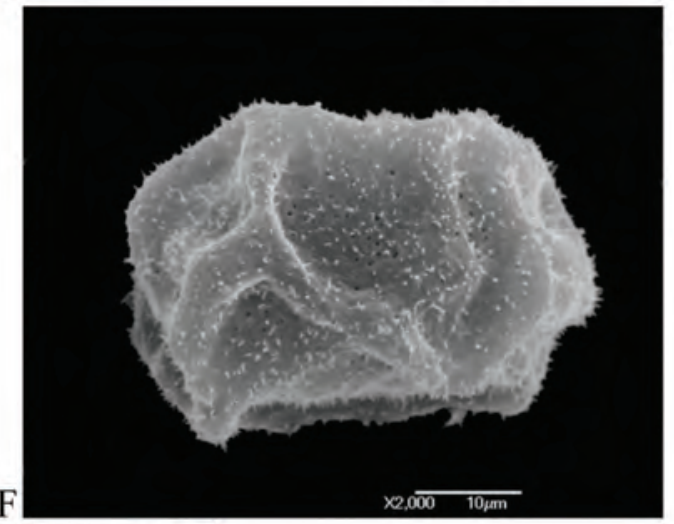

$\mathrm{F}$

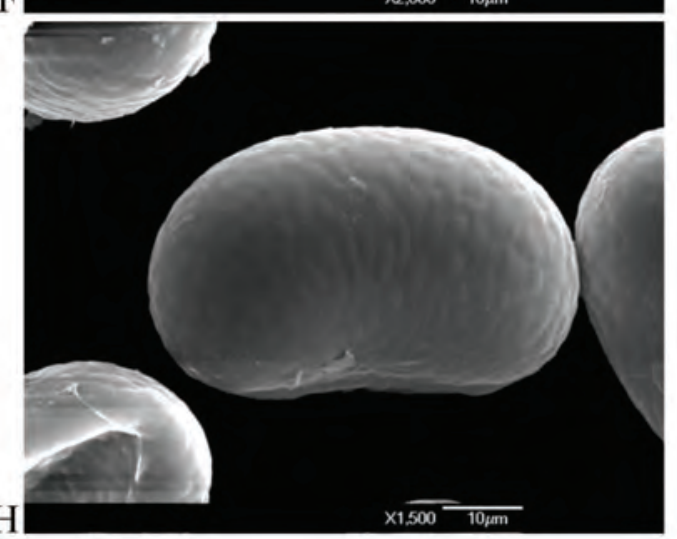

Figura 7. Dryopteridaceae (cont.): Phanerophlebia remotispora, A: vista ecuatorial, MO, B: vista ecuatorial, MEB; Polystichum turrialbae, C: vista ecuatorial, MO, D: vista distal, MEB; Tectariaceae: Tectaria heracleifolia, E: vista ecuatorial, MO, F: vista ecuatorial, MEB;

Polypodiaceae: Campyloneurum angustifolium, G: vista proximal, MO, H: vista ecuatorial, MEB. 

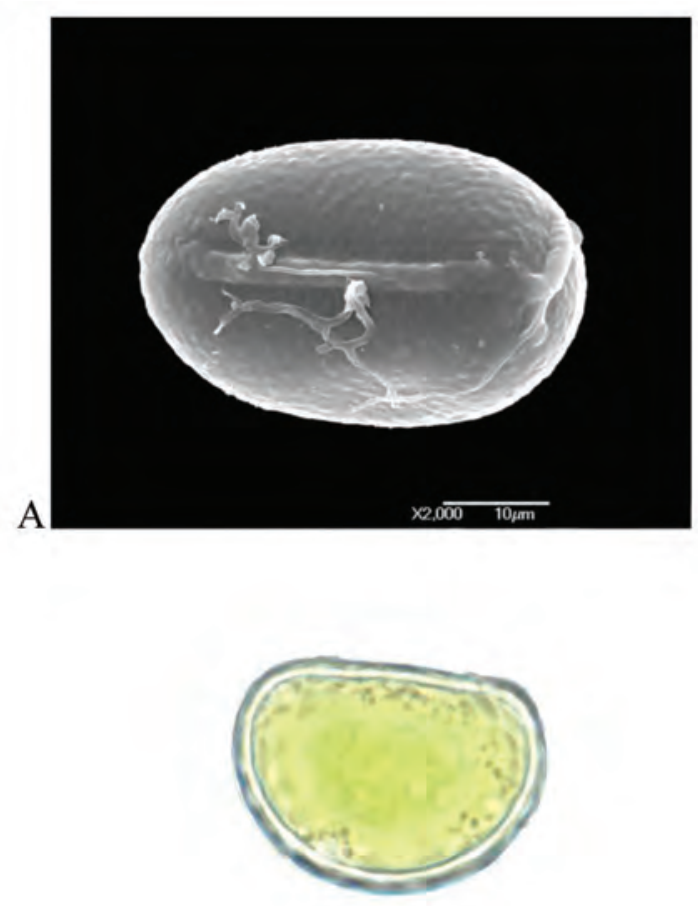

C

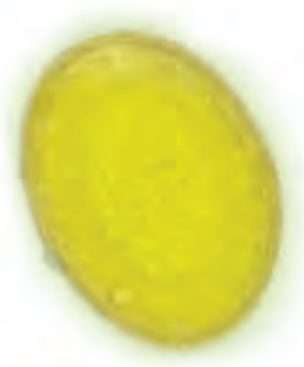

$\mathrm{E}$

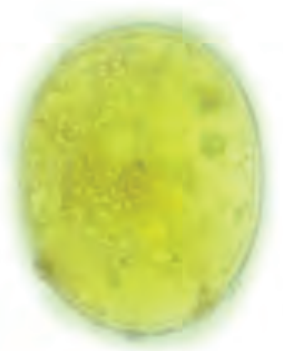

G
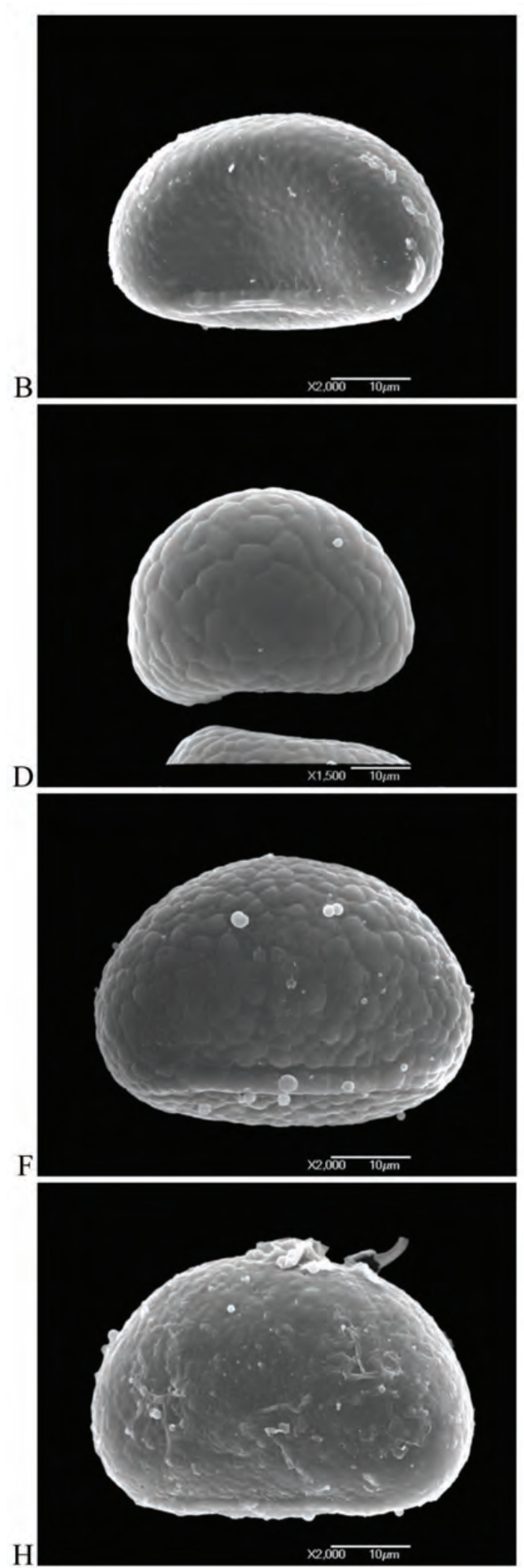

Figura 8. Polypodiaceae (cont.): Campyloneurum xalapense, A: vista proximal, MEB, B: vista ecuatorial, MEB; Pecluma sursumcurrens, C: vista ecuatorial, MO, D: vista ecuatorial, MEB; Pleopeltis crassinervata, E: vista distal, MO, F: vista ecuatorial, MEB; Polypodium furfuraceum, G: vista distal, MO, H: vista ecuatorial, MEB. 


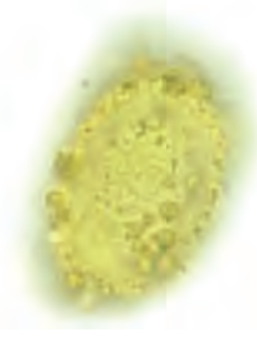

A

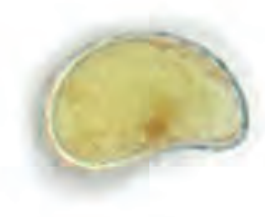

C

E

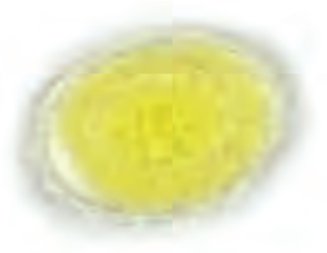

G

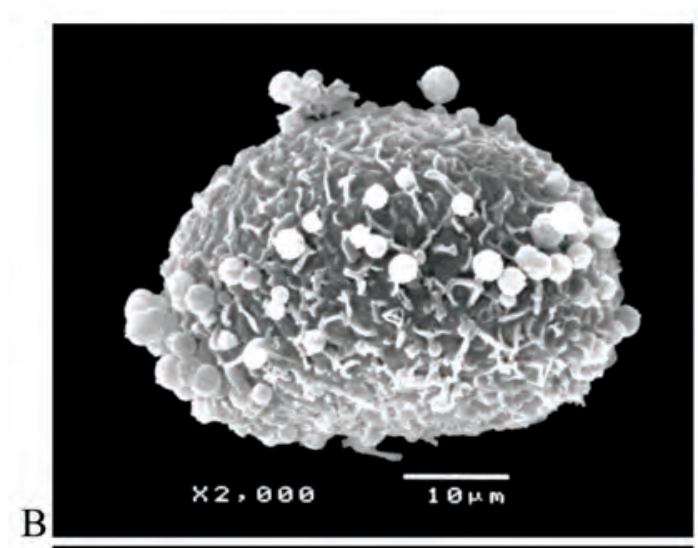

B

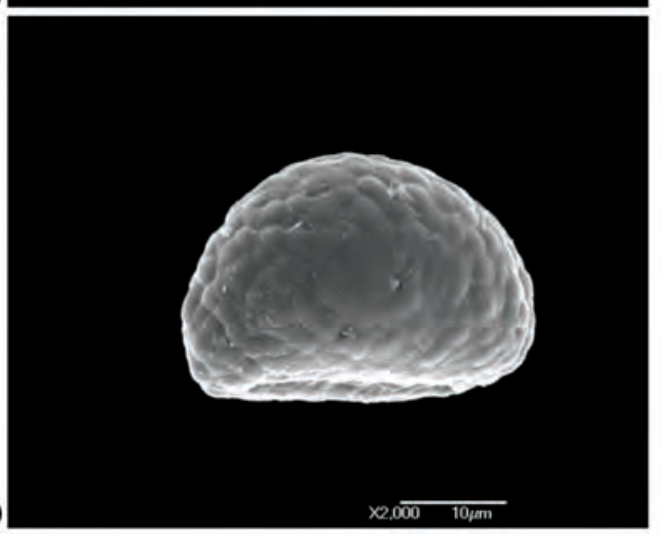

D

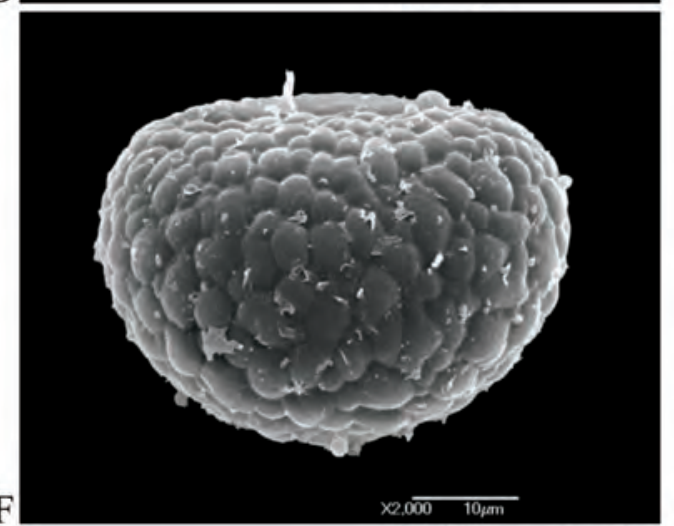

F

$\times 2,0 \overline{00 \quad 10 \mathrm{mam}}$

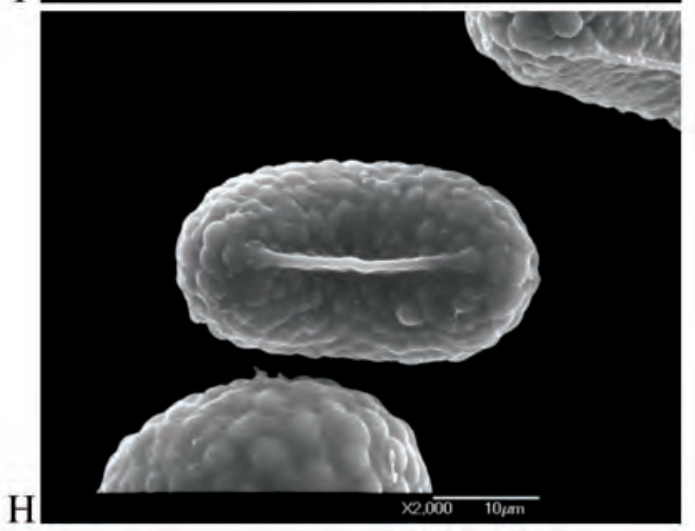

Figura 9. Polypodiaceae (cont.): Polypodium lepidotrichum, A: vista distal, MO, B: vista ecuatorial, MEB; P. longepinnulatum, C: vista ecuatorial, MO, D: vista ecuatorial, MEB; P. plebeium, E: vista distal, MO, F: vista ecuatorial, MEB; P. plesiosorum, G: vista ecuatorial, MO, H: vista proximal, MEB. 
A

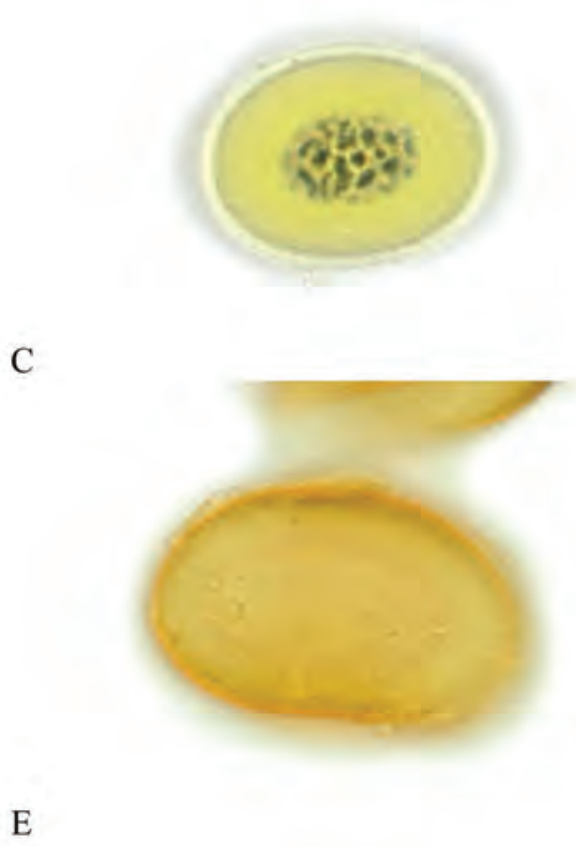

$\mathrm{C}$

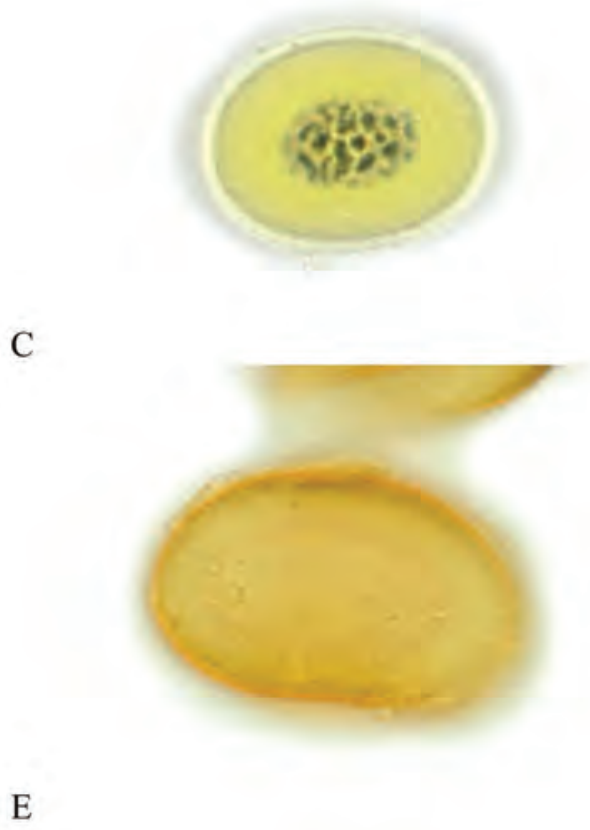

$\mathrm{E}$

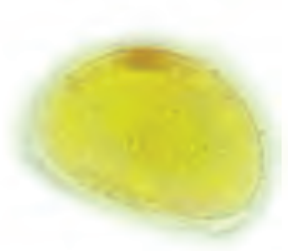

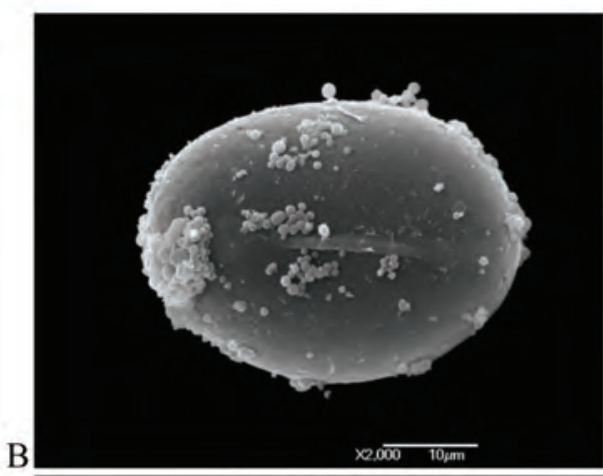

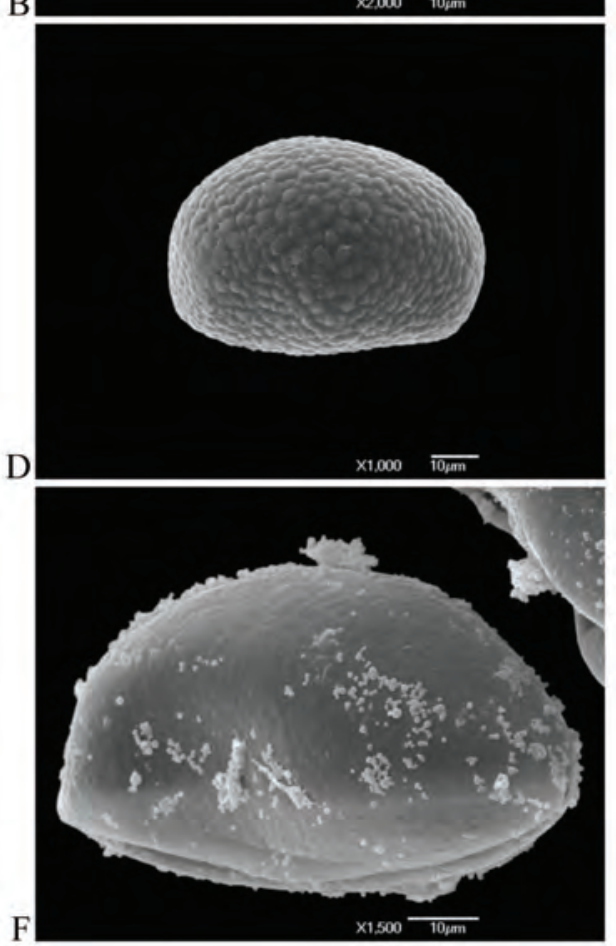

Figura 10. Polypodiaceae (cont.): Polypodium polypodioides var. aciculare, A: vista ecuatorial, MO, B: vista proximal, MEB; P. rhodopleuron, C: vista distal con análisis L-O en contraste de fases, MO, D: vista ecuatorial, MEB; P. villagranii, E: vista distal, MO, F: vista ecuatorial, MEB.

amarillo, EP: 23.5 (28) $31.6 \pm 1.80 \mu \mathrm{m}$, EE: 45 (52) $58 \pm 3.40 \mu \mathrm{m}, \mathrm{P} / \mathrm{E}: 0.54$ oblado, Ex: $0.98(2.2) 2.94 \pm 0.62 \mu \mathrm{m}$, O: isopolar, verrugada. Ejemplares: FGN 234, FGN 275.

\section{Polypodium polypodioides (L.) Watt var. aciculare Weath.}

Figura 10A, B

Espora monolete, VP: elipsoidal, VE: reniforme, Color: amarillo, EP: 29 (33) $37 \pm 2.46 \mu \mathrm{m}$, EE: 39 (44) $52 \pm 3.57 \mu \mathrm{m}$, P/E. 0.74 oblado, Ex: 1 (1.1) $2 \pm 0.40 \mu \mathrm{m}$, O: isopolar, ligeramente verrugada, con glóbulos escasos en su superficie. Ejemplar: FGN 290.

\section{Polypodium rhodopleuron Kunze}

Figura 10C, D Espora monolete, VP: elipsoidal, VE: reniforme, Color: ama- rillo, EP: 48 (51) $54 \pm 2.00 \mu \mathrm{m}$, EE: 62 (69) $72 \pm 2.53 \mu \mathrm{m}$, P/E. 0.74 oblado, Ex: 3 (3.7) $4.9 \pm 0.60 \mu \mathrm{m}$, O: isopolar, verrugada, en contraste de fases se observa que éstas están constituidas por gránulos. Ejemplares: FGN 228, NVP 1.

Polypodium villagranii Copel.

Figura 10E, F Espora monolete, VP: elipsoidal, VE: reniforme, Color: pardo claro, EP: 46 (56) $66 \pm 4.56 \mu \mathrm{m}$, EE: 82 (87) $99 \pm 6.73 \mu \mathrm{m}$, P/E. 0.65 oblado, Ex: 1 (2.5) $4 \pm 0.74 \mu \mathrm{m}, \mathrm{O}$ : isopolar, verrugada con glóbulos más abundantes en el eje ecuatorial menor. Ejemplar: FGN 291. 
Clave para la identificación de esporas de las especies de helechos y licofitas de Río Malila, Hidalgo, México

1a. Esporas aletes, la lesura no evidente, eláteres presentes o caducos, esporas clorofílicas con ornamentación psilada

Equisetum myriochaetum

1b. Esporas triletes o monoletes, la lesura evidente, sin eláteres presentes, esporas no clorofílicas, o si clorofílicas entonces con ornamentación reticulada-ornada

2a. Esporas triletes, tétrada tetrahédrica, simetría radial

3a. Esporas con un cíngulo ecuatorial evidente

4a. Ornamentación distal foveolada, esporas hialinas a amarillas claras

Lophosoria quadripinnata

4b. Ornamentación distal hamulosa, esporas rojizas Pteris quadriaurita

3b. Esporas sin un cíngulo ecuatorial evidente

5a. Esporas triangular-globosas a esféricas

6a. Esporas pardo claras, ornamentación verrugada a fosulada

Cheilanthes notholaenoides

6b. Esporas pardo oscuras, ornamentación ruguladacrestada

Mildella intramarginalis

5b. Esporas triangulares, convexas o cóncavas, no globosas, o si globosas con presencia de crestas

7a. Esporas de 15 a $30 \mu \mathrm{m}$, amarillas a anaranjadas con ornamentación clavada o equinulada

8a. Esporas con clavas presentes, evidentes, de 1 a $4 \mu \mathrm{m}$ de largo

Selaginella pallescens

8b. Esporas sin clavas presentes, con espínulas u otra estructura en su superficie

Selaginella stenophylla

7b. Esporas mayores a $30 \mu \mathrm{m}$, anaranjadas o pardas claras, con ornamentación papilada o rugulado-crestada

9a. Esporas con ornamentación papilada a gemada 10a. Esporas isopolares con gemas uniformes Llavea cordifolia

10b. Esporas heteropolares, ornamentación proximalmente papila y distalmente con diversos engrosamientos de $2.5 \mu \mathrm{m}$

Dennstaedtia globulifera

9b. Esporas con ornamentación rugulado-crestada o microgranular

11a. Esporas triangular-convexas, pardas, con ornamentación rugulado-crestada

Pellaea ovata

11b. Esporas triangulares, pardas claras, con ornamentación rugulada, de apariencia microgranular Adiantum andicola

2b. Esporas monoletes, tétrada tetragonal, simetría bilateral 12a. Esporas con perisporio claramente visible en MO; mayor a $1 \mu \mathrm{m}$ de grosor, holgadamente rodeando el exosporio, o a más de $2 \mu \mathrm{m}$ de éste

13a. Perisporio liso en corte óptico, sin crestas ni espinas

14a. Perisporio foveolado, al menos en algunas partes, y con bordes que forman patrones circulares o hexagonales, de color pardo obscuro

Thelypteris ovata var. lindheimeri

14b. Perisporio no foveolado, plegado de forma irregular y sin formar estructuras circulares, de color pardo claro

15a. Perisporio con 4 a 7 pliegues, en corte óptico se observa que el perisporio es paralelo al exosporio en la mayor parte de la espora, este último equinado

Diplazium lonchophyllum

15b. Perisporio presentando más de 7 pliegues, el borde ondulado, nunca recto ni paralelo al exosporio, o sólo en 2/3 del exosporio, éste sin espinas

16a. El perisporio con pliegues anchos que dan apariencia ondulada en corte óptico, esporas de $30.5 \pm 3.34 \times 40 \pm 2.79 \mu \mathrm{m}$

Phanerophlebia remotispora

16b. El perisporio con pliegues estrechos que dan apariencia ondulada en corte óptico y en algunas partes paralelo al exosporio, esporas de $34 \pm 2.54 \times 43 \pm 2.99 \mu \mathrm{m}$

Elaphoglossum obscurum

13b. Perisporio con crestas o espinas

17a. Espora con perisporio foveolado a reticulado

18a. Esporas con perisporio foveolado Tectaria heracleifolia

18b. Esporas con perisporio reticulado 19a. Esporas con retículo ornado, clorofílicas

Onocleopsis hintonii

19b. Esporas con el retículo no ornado, no clorofílicas

20a. Pliegues del perisporio rectos, espora con apariencia trapezoidal

Holodyctium ghiesbreghtii

20b. Pliegues del perisporio ondulados, no rectos; espora elipsoidal o irregular 21a. Espora con perisporio, aladas evidentes y anchas; retículo con numerosas papilas que conectan con el exosporio

Asplenium monanthes

21b. Espora sin alas aparentes, pliegues romos y cortos, retículo simple, sin papilas que conecten con el exosporio

Polystichum turrialbae 
17b. Espora con perisporio, equinado, espinulado o microcrestado

22a. Perisporio no alado, con ornamentación microvermiculada a microcrestada en toda su superficie

Woodwardia martinezii

22b. Perisporio alado, con muri formando un patrón reticulado a vermiculado, espinas o báculas entre las alas 23a. Espacio entre las alas reticulado, con báculas o espinas, muri no foveolados en la base

Asplenium sessilifolium var. sessilifolium 23b. Espacio entre las alas con escasas espinas, muri foveolados en la base Asplenium abscissum

12b. Esporas sin perisporio claramente visible en MO; menor a $1 \mu \mathrm{m}$ de grosor, no se observa holgadamente rodeando el exosporio, ni se encuentra extendido más de $1 \mu \mathrm{m}$ del exosporio

24a. Esporas con ornamentación equinada

25a. Espinas de 3 a $7 \mu \mathrm{m}$ de largo, foveoladas en la base, esporas obladas, de color pardo oscuro

Cystopteris fragilis 25b. Espinas no foveoladas en su base, escasamente foveoladas en la parte media; de 3.5 a $9.8 \mu \mathrm{m}$ de largo, esporas subobladas

Ctenitis equestris

24b. Esporas con ornamentación verrugada, psilada, fosulada, clavada o papilada, nunca equinada

26a. Exosporio de $2 \mu \mathrm{m}$ o más de grosor

27a. Esporas hamulosas, con espinas de hasta $4.5 \mu \mathrm{m}$ de largo

Macrothelypteris torresiana

27b. Esporas verrugadas o psiladas, no hamulosas

28a. Esporas conspicuamente verrugadas en corte óptico o psiladas 29a. Esporas con exosporio psilado, con gránulos en su superficie Polypodium furfuraceum

29b. Esporas psiladas a verrugadas; sin gránulos en su superficie 30a. Esporas psiladas a ligeramente verrugadas, hialinas a amarillas claras

31a. Esporas amarilla traslúcida, de $34 \pm 1.96 \times 58.5 \pm 1.92 \mu \mathrm{m}$, verrugas de $5 \mu \mathrm{m}$ de diámetro
Campyloneurum angustifolium 31 b. Esporas amarilla traslúcida de $40 \pm 4.56 \times 63 \pm 6.75 \mu \mathrm{m}$, verrugas de $2 \mu \mathrm{m}$ de diámetro

Campyloneurum xalapense 30b. Esporas ligeramente verrugadas, amarillas

32a. Esporas elipsoidales, de 23(28) $31 \times 45(51) 58 \mu \mathrm{m}$

Polypodium plesiosorum

32b. Esporas elipsoidal-globosas, de 27(30)33 × 39(43) $48 \mu \mathrm{m}$

Polypodium plebeium

28b. Esporas verrugadas, aunque no de manera conspicua en corte óptico, fosuladas o psiladas

33a. Esporas verrugadas, las

verrugas de apariencia granular al ser observadas en contraste de fases

Polypodium rhodopleuron 33b. Esporas verrugadas, sin apariencia granular en contraste de fases; psiladas o vermiculadas

34a. Esporas vermiculadas, con glóbulos, papilas y báculas

Polypodium lepidotrichum 34b. Esporas verrugadas 35a. Esporas pardas, reniformes en vista ecuatorial, ornamentación tenuemente verrugada, con glóbulos en su superficie, exina parda

Polypodium villagranii

35b. Esporas amarillas, no reniformes en vista ecuatorial, sin glóbulos en su superficie, las verrugas de apariencia más o menos hexagonal, exina hialina

Pecluma sursumcurrens

26b. Exosporio de menos de $2 \mu \mathrm{m}$ de grosor

36a. Esporas psiladas, amarillas, con escasos glóbulos en la superficie Polypodium polypodioides var. aciculare 36b. Esporas verrugadas o fosuladas, amarillas a pardas claras u obscuras, sin glóbulos en su superficie 
37a. Esporas de color pardo oscuro a claro, de ornamentación fosulada Blechnum appendiculatum 37b. Esporas de color amarillo a pardo claro, de ornamentación verrugada 38a. Esporas amarilloclaras, verrugadas, las verrugas de distinto tamaño, las más grandes en la cara distal, exosporio hialino a amarillo claro

Polypodium longepinnulatum 38b. Esporas amarillas, verrugadas, verrugas de tamaño uniformes, exosporio pardo

Pleopeltis crassinervata

\section{Discusión}

Las características de las esporas, tales como la ornamentación, el color, la forma, el tamaño, el exosporio, el perisporio y el tipo de lesura, entre otras, permiten identificar adecuadamente el taxon al que pertenecen, incluso en el caso de especies y géneros afines como Pleopeltis, Pecluma y Polypodium, de la familia Polypodiaceae, en donde predomina la ornamentación verrugada. La mayoría de las características de las esporas estudiadas concuerda con lo reportado en trabajos previos (Erdtman y Sorsa, 1971; Tryon y Tryon, 1982; Tryon y Lugardon, 1991); incluso las particularidades de las esporas descritas por vez primera (Ctenitis equestris, Elaphoglossum obscurum, Pecluma sursumcurrens y Polystichum turrialbae) son consistentes con lo citado para las esporas de sus respectivos géneros (Erdtman y Sorsa,1971; Tryon y Tryon,1982; Tryon y Lugardon, 1991; Arreguín-Sánchez et al., 2004), al igual que para con otras especies, p. ej. las esporas de Polystichum turrialbae poseen un perisporio crestado y plegado, similar al mencionado para las esporas de $P$. giganteum y $P$. montevidense por Kessler et al. (2005).

Sólo en Cystopteris fragilis se encontraron algunas diferencias importantes en las descripciones de las esporas, mientras en el presente estudio se observó con espinas; en algunas descripciones se mencionan con ornamentación verrugada (Tryon y Tryon, 1982; Arreguín-Sánchez et al., 1996). Palinológicamente esta especie está ampliamente estudiada, primeramente por Hagenah (1961), Kaur (1963), Erdtman y Sorsa (1971), Belling y Heusser (1974), Pearman (1976), Murillo y Bless (1978), posteriormente por Dyer et al. (2000); y está muy relacionada con C. dickieana R.Sim, de la cual únicamente difiere por la ornamentación de la espora; equinada en $C$. fragilis y verrugada en $C$. dickieana (Höran- dl, 1989). Dada la dificultad para distinguir ambas especies, es probable que $C$. dickieana se encuentre en México, pero se necesita una revisión amplia para poder asegurarlo.

Aun cuando las dimensiones reportadas en las especies estudiadas varían con relación al tamaño mencionado para las esporas en diferentes obras, probablemente esta diferencia se relaciona con la técnica utilizada, como ya lo mencionó Prada (1990). En algunas especies como Campyloneurum xalapense, Lophosoria quadripinnata, Onocleopsis hintonii, Pellaea ovata, Polypodium villagranii, Pteris quadriaurita y Woodwardia martinezii se observó la mayor variación de tamaños, que según Large y Braggins (1991), es posible que se relacione con la ploidía, pues es sabido que ésta puede variar entre diferentes poblaciones de la misma especie. De estos taxa es necesario remarcar a Lophosoria quadripinnata y a Woodwardia martinezii, en las cuales se encontraron esporas con un tamaño mucho mayor a lo reportado en otros estudios. Por ejemplo, L. quadripinnata mostró esporas de $81 \times 74 \mu \mathrm{m}$, con un mínimo de 63 y máximo de $107 \mu \mathrm{m}$, mientras en distintas fuentes se registraron tamaños de $37 \times$ $54 \mu \mathrm{m}, 32 \times 48 \mu \mathrm{m}$ (Erdtman y Sorsa, 1971), 41 a $53 \mu \mathrm{m}$ (Arreguín-Sánchez et al., 1996), $56 \times 50 \mu \mathrm{m}$ (Pérez-García et al., 1995) y de 33 a $89 \mu \mathrm{m}$ (Contreras-Duarte et al., 2006). De igual manera, para W. martinezii se encontraron esporas de $78 \times 54 \mu \mathrm{m}$ con mínimo de 29 y máximo de 90 $\mu \mathrm{m}$, mientras sólo se habían descrito tamaños de $51 \times 76$ $\times 52 \mu \mathrm{m}$ (Erdtman y Sorsa, op cit.). En L. quadripinnata no existen antecedentes de poliploidía ni apogamia que pudieran explicar la variación de tamaño de sus esporas, no así en W. martinezii, para la cual Klekowski (1969) reporta apogamia.

$\mathrm{Al}$ contar con un mejor conocimiento palinológico de las especies de helechos mexicanos será posible, como en el caso del polen de las angiospermas, aportar datos sobre el impacto de los cambios ambientales en la vegetación durante las últimas décadas (Sánchez-Dzib et al., 2009). Además, esta información resulta indispensable para la realización de estudios de dispersión y lluvia de esporas, pues sólo a partir de la adecuada caracterización específica de estas partículas biológicas se puede determinar la estacionalidad o fenología de esporulación en este grupo de plantas; tema poco abordado para los helechos y licofitas. El presente trabajo aporta datos para la correcta identificación de los taxa locales, los cuales, indudablemente serán de utilidad para futuros muestreos aerobiológicos en el sitio de estudio.

\section{Agradecimientos}

Se agradece el apoyo del CONACYT mediante la beca (224680) otorgada al primer autor, igualmente al programa de la Maestría en Biología de la Universidad Autónoma Metropolitana Unidad Iztapalapa. Reconocemos la valiosa asesoría palinológica de Enrique Martínez Hernández y de Elia Ramírez Arriaga, del Instituto de Geología de la UNAM y 
de Ana Rosa López Ferrari, encargada del Herbario Metropolitano "Dr. Ramón Riba y Nava Esparza" de la UAM- Iztapalapa, así como a Silvia Espinosa Matías del Laboratorio de Microscopía Electrónica de la Facultad de Ciencias de la UNAM, quien se encargó del procesamiento de las muestras y de la obtención de imágenes digitales en el MEB. Finalmente agradecemos los valiosos comentarios y sugerencias de Gabriela E. Giudice y de un revisor anónimo, que enriquecieron el presente trabajo.

\section{Literatura citada}

Arreguín-Sánchez M.L. 1985. Morfología de las esporas de la subfamilia Asplenoideae del Valle de México. En: Montúfar A. Coord. Estudios Palinológicos y Paleobotánicos, pp. 7-22, Instituto Nacional de Antropología e Historia, México, D.F.

Arreguín-Sánchez M.L. 1988. Morfología de las esporas de la familia Selaginellaceae del valle de México. Palynologica et Paleobotanica 1:5-18.

Arreguín-Sánchez M.L. 1991. Claves para la separación de los taxa de pteridofitas del Valle de México por medio de sus esporas. Palynologica et Paleobotanica 3:5-54.

Arreguín-Sánchez M.L. y Aguirre-Claverán R. 1990. Morfología de las esporas isospóreas triletes de pteridofitas de Nuevo León, México. Palynologica et Paleobotanica 2:5-62.

Arreguín-Sánchez M.L., Fernández-Nava R., Palacios-Chávez R. y Quiroz-García D.L. 1996. Morfología de las esporas isospóreas del estado de Querétaro, México. Polibotánica 2:10-60.

Arreguín-Sánchez M.L., Fernández-Nava R. y Quiroz García D.L. 2004. Pteridoflora del Valle de México. Escuela Nacional de Ciencias Biológicas del Instituto Politécnico Nacional, México, D.F.

Belling A.J. y Heusser C.J. 1974. Spore morphology of the Polypodiaceae of northeastern north America. I. Bulletin of the Torrey Botanical Club 101:326-339.

Benito-Rica V. y Soto-Torres J. 2001. Polinosis y aerobiología del polen en la atmósfera de Santander. Alergologia e Inmunología Clínica 16:84-90.

Britton D.M. y Jeremy A.C. 1974. The spores of Dryopteris filixmas and related taxa in North America. Canadian Journal of Botany 52:1923-1926.

Chew F.T., Lim S.H., Shang H.S., Siti-Dahlia M.D., Goh D.Y.T., Lee B.W., Tan H.T.W. y Tan T.K. 2000. Evaluation of allergenicity of tropical pollen and airborne spores in Singapore. Allergy 55:340-347.

Ching O.T. 2004. Aerobiology, image analysis and allergenicity of pollen and spores in Singapore. Tesis de doctorado, Department of Pediatrics, National University of Singapore, Singapore. $235 \mathrm{pp}$.

Christenhusz M.J.M., Zhang X.C. y Schneider H. 2011. A linear sequence of extant families and genera of Lycophytes and Ferns. Phytotaxa 19:7-54.

Contreras-Duarte A.R., Bogotá-Ángel R.G. y Jiménez-Bulla L.C. 2006. Atlas de las esporas de pteridófitos de Chipaque (Cundinamarca, Colombia). Caldasia 28:327-357.

Dyer A.F., Parks J.C. y Lindsay S. 2000. Historical review of the uncertain taxonomic status of Cystopteris dickieana R.Sim (Dickie's bladder fern). Edinburgh Journal of Botany 57:71-81.

Erdtman G. 1960. The acetolisis method, a revised descriptions.
Svensk Botanisk Tidskrift utgifven af Svenska Botaniska Föreningen 54:561-564.

Erdtman G. y Sorsa P. 1971. Pollen and Spore Morphology, Plant Taxonomy: Pteridophyta (Text and Additional Illustrations). Almqvist \& Wiksells, Estocolmo.

Gastony G.J. 1979. Spore morphology in the Cyatheaceae. III. The genus Trichipteris. American Journal of Botany 66:1238-1260.

Giudice G.E., Morbelli M.A. Piñeiro M.R., Copello M. y Erra G. 2004. Spore morphology of the Polypodiaceae from the northwest Argentina. Amercian Fern Journal 94:9-27.

Giudice G.E., Morbelli M.A., Macluf C.C., Hernández M. y Ruiz A. 2006. Morphology and ultrastructure of the spores of Dennstaedtiaceae from North West Argentina. Review of Paleobotany and Palynology 141:245-257.

Hagenah D.J. 1961. Spore studies in the genus Cystopteris I. the distribution of Cystopteris with non-spiny spores in North America. Rhodora 63:181-193.

Hörandl E. 1989. Cystopteris dickieana R. Sim (Pteridophyta) neu für Österrich und andere Gebeite. Verhandlungen der Zoologisch-Botanischen Gesellschaft in Österreich 126:179-183.

Kaur S. 1963. The gametophyte of Cystopteris fragilis. Proceedings of the Indian Academy of Sciences - Section B 58:148-152.

Kessler M., Smith A.R. y Sundue M. 2005. Notes on the genus Polystichum (Dryopteridaceae) in Bolivia, with descriptions of ten new species. Brittonia 57:205-227.

Klekowski E.J. 1969. Reproductive biology of the pteridophyta. III. A study of the Blechnaceae. Botanical Journal of the Linnean Society 62:361-377.

Kremp G.O.W. 1965. Morphologic Encyclopedia of Palynology, An International Collection of Definitions and Illustrations of Spores and Pollen. The University of Arizona Press, Tucson.

Lacey M.E. y McCartney H.A. 1994. Measurement of airborne concentrations of spores of bracken (Pteridium aquilinum). Grana 33:91-93.

Large M.F. y Braggins J.E. 1991. Spore Atlas of New Zealand Ferns and Fern Allies. SIR Publishing, Wellington.

Lellinger D.B. 2002. A Modern Multilingual Glossary for Taxonomic Pteridology. Pteridologia 3. American Fern Society, Washington, DC.

Lorea F. y Riba R. 1990. Guía para la Recolección y Preparación de Ejemplares para Herbario de Pteridofitas. Consejo Nacional de la Flora de México A.C./Universidad Nacional Autónoma de México, México, D.F.

Lugardon B. 1986. Données ultrastructurales sur la fonction de l'exospore chez les Ptéridophytes (Ultrastructural data of the exospore function in pteridophyte spores). En: Blackmore S., Ferguson I.K. y Linnean Society of London Eds. Pollen and Spores: Form and Function, pp. 251-264, Academic Press, Londres.

Mendoza-Ruiz A.C. 2001. Morfogénesis de la fase sexual de pteridofitas mexicanas, familia Dryopteridaceae. Tesis de Maestría, Facultad de Ciencias, Universidad Nacional Autónoma de México, México, D.F. 87 pp.

Mendoza-Ruiz A. y Pérez-García B. 2009. Morphogenesis of the gametophytes of eight mexican species of Blechnum (Blechnaceae). Acta Botanica Mexicana 88:59-72.

Mickel J.T. y Smith A.R. 2004. The Pteridophytes of Mexico. New York Botanical Garden Press, Nueva York.

Morbelli M.A. 1976. Estudio palinológico de las especies Austrosudamericanas del género Blechnum L., subgénero Blechnum 
(Blechnaceae-Pteridophyta). Boletín de la Sociedad Argentina de Botánica 17:155-175.

Morbelli M.A. y Giudice E.G. 2010. Spore wall ultrastructure of Polypodiaceae fron north-west Argentina. Grana 49:204-214.

Morbelli M.A. y Michelena I.G. 1989. Palynological analysis of Cheilanthes species (Adiantaceae-Pteridophyta) of northwestern Argentina. Grana 28:295-304.

Murillo M.T. y Bless M.J.M. 1978. Spores of recent Colombian pteridophyta. II. Monolete spores. Review of Paleobotany and Palynology 25:319-365.

Pangua E. y Prada C. 1988. Tipos esporales en Aspleniaceas Ibéricas. Lagascalia 15 (extra):157-167.

Pearman R.W. 1976. A scanning electron microscope investigation of the spores of the genus Cystopteris. Fern Gazette 11:221-230.

Pérez-García B. y Mendoza A. 2005. Comparative studies of the gametophytes of five new world species of Tectaria (Tectariaceae). American Fern Journal 95:141-152.

Pérez-García B. 1988. Morfogénesis de gametofitos de Cyatheaceae (Pteridophyta: Filicales) Tesis de Doctorado, Facultad de Ciencias, Universidad Nacional Autónoma de México. 224 pp.

Pérez-García B., Mendoza-Ruiz A., Espinosa-Matías S. y GómezPignataro L.D. 2010. Gametohpyte morphology of Platycerium andinum Baker and Platycerium wandae Racif. Micron 41:806-813.

Pérez-García B., Fraile M.E. y Mendoza A. 1995. Desarrollo del gametofito de Lophosoria quadripinnata (Filicales: Lophosoriaceae). Revista de Biología Tropical 43:55-60.

Prada C. 1990. El papel de la palinología en los estudios actuales sobre pteridófitos. En: Rita J. Ed. Taxonomía, Biogeografía y Conservación de Pteridófitos, pp. 39-46, Institut Menorqui d'Estudis/Societat d'Història Natural de les Bealers, Mallorca.

Punt W., Hoen P.P., Blackmore S., Nilsson S. y Le Thomas A. 2007. Glossary of pollen and spore terminology. Review Paleobotany and Palynology 143:1-81.

Ramírez-Cruz S., Sánchez-González A. y Tejero-Díez D. 2009.
La Pteridoflora del Parque Nacional Los Mármoles, Hidalgo, México. Boletín de la Sociedad Botánica de México 84:35-44.

Ramírez-Trejo M.R. 2002. Los bancos de esporas de helechos en diferentes suelos y tipos de vegetación del estado de Hidalgo. Tesis de Maestría, Facultad de Ciencias, Universidad Nacional Autónoma de México, México, D.F. 67pp.

Sánchez-Dzib Y.A., Sosa-Nájera S. y Lozano-García M.S. 2009. Morfología polínica de especies de la selva mediana subperennifolia en la cuenca del río Candelaria, Campeche. Boletín de la Sociedad Botánica de México 84:83-104.

Smith A.R., Pryer K.M., Schuettpelz E., Korall P., Schneider H. y Wolf. P.G. 2006. A classification for extant Ferns. Taxon 55:705-731.

Tryon A.F. 1986. Stasis, diversity and function in spores based on an electron microscope survey of the Pteridophyta. En: Blackmore S., Ferguson I.K. y Linnean Society of London Eds. Pollen and Spores: Form and Function, pp. 233-249, Academic Press, Londres.

Tryon A.F. y Lugardon B. 1991. Spores of the Pteridophyta: Surface, Wall Structure and Diversity Based on Electron Microscope Studies. Springer-Verlag, Nueva York.

Tryon R.M. y Tryon A.F. 1982. Fern and Fern Allied Plants with Special Reference to Tropical America. Springer-Verlag, Nueva York.

Tschudy R.H. y Tschudy B.D. 1965. Modern fern spores of Rancho Grande, Venezuela. Acta Botanica Venezuelica 1:9-70.

Uhera K. y Kurita S. 1986. Ultrastructure of sporoderm morphogenesis in Psilotum nudum (L.) Griseb. Science Reports of the Research Institute of Evolutionary Biology 3:171-181.

Wood C.C. 1973. Spore variation in Thelypteridaceae. En: Jeremy A.C., Crabbe J.A. y Thomas B.A. Eds. The Phylogeny and Classification of the Ferns, pp. 191-201, Academic Press, Londres.

Recibido: 14 de marzo de 2012

Aceptado: 28 de mayo de 2012 